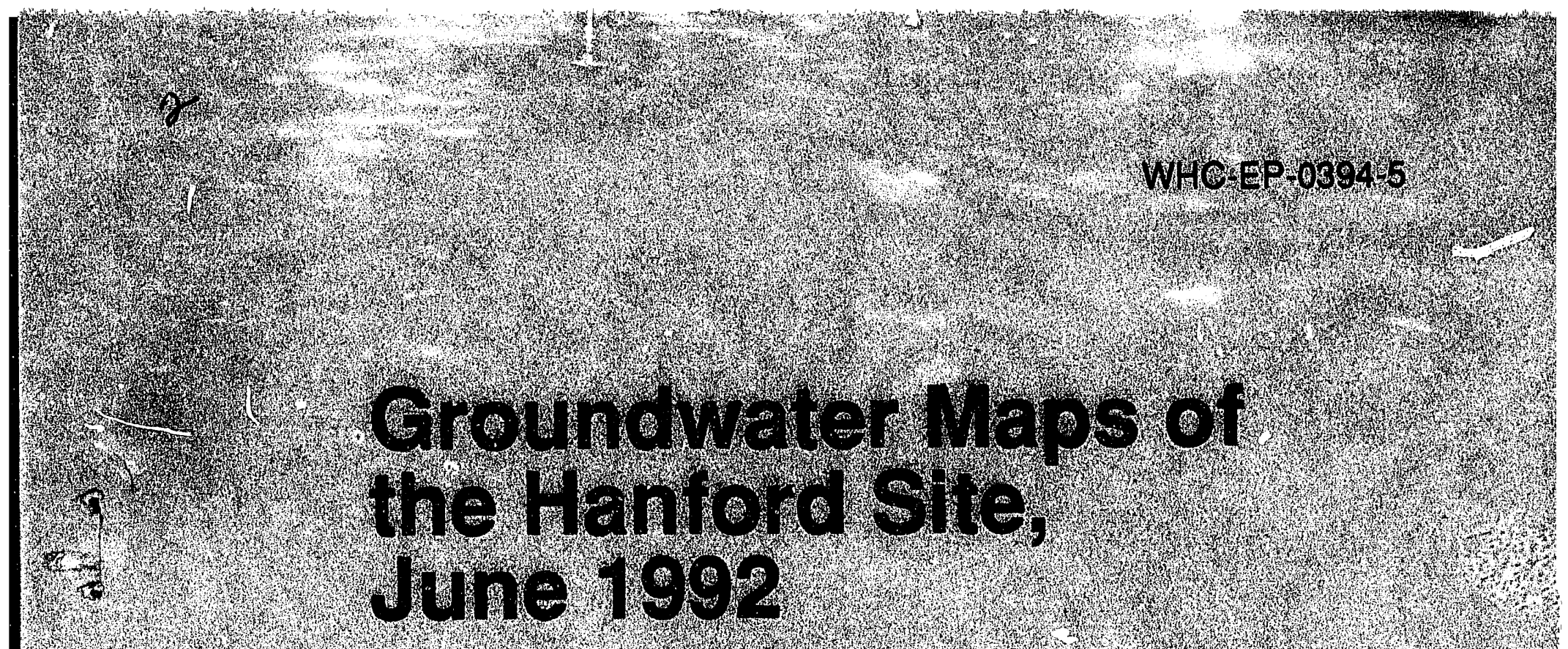

Prepared for the USS Department of Energy Orfice of Environmental bestoration ahd Waste Mranagement

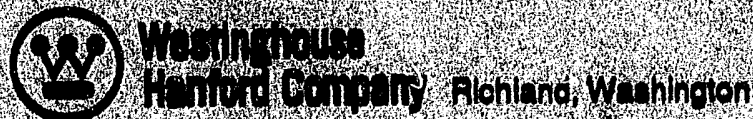

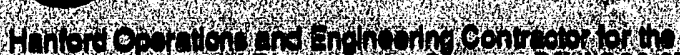

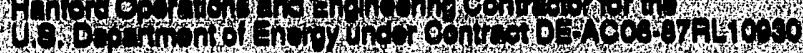

RECEIVED JUL O \& 1993 ost

Approved tor Public Reloaso 


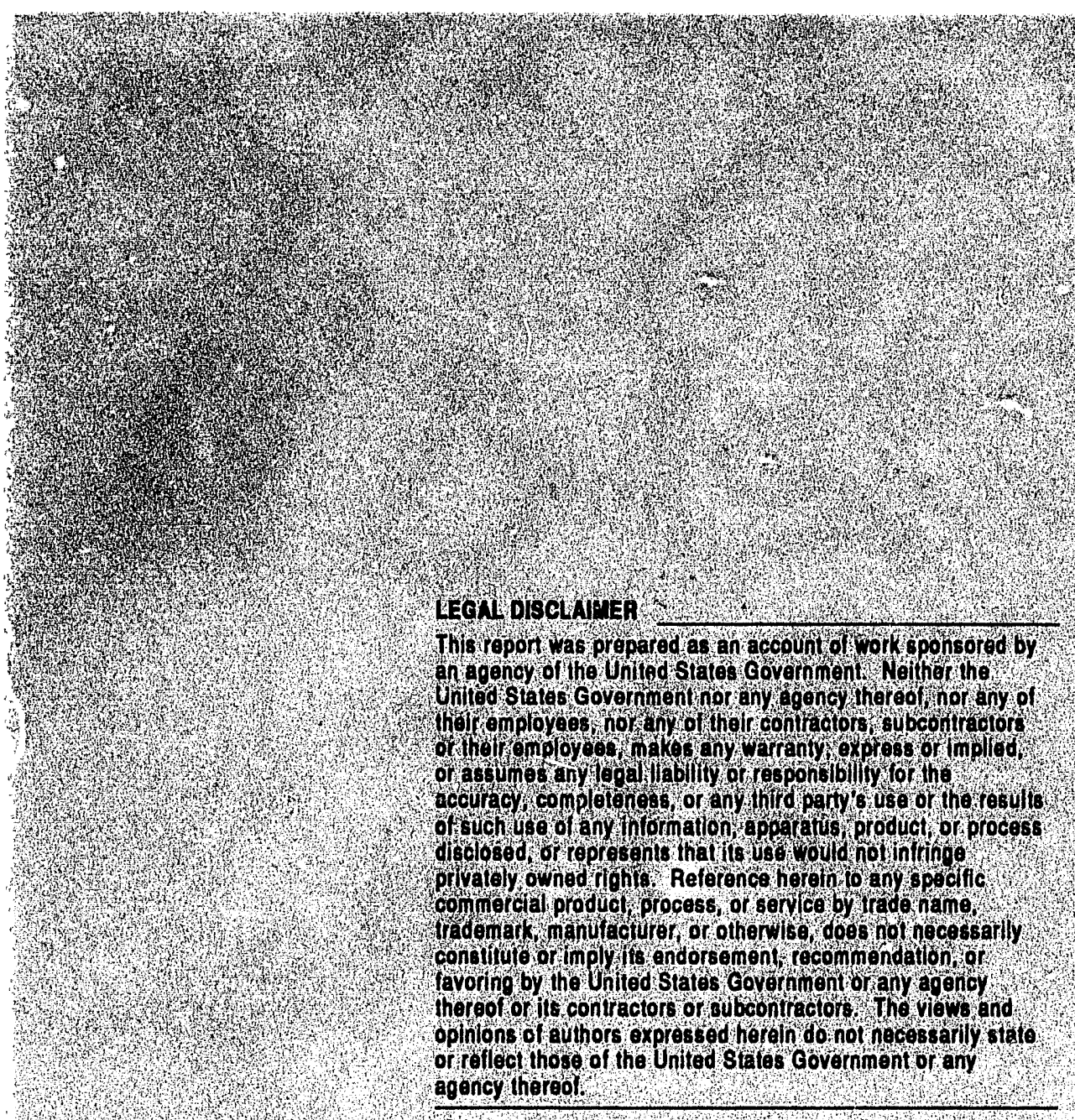

This report has been reproduced from the best avallable copy. Available in paper copy and microticho.

\section{Avaliable to the U. Department of Energy}

and its contractors trom

Ofilce of Sojentilio and Technical Informatlon

P.O. Box 62

6ak Ridur o $1 \mathrm{~N} 3783$

(615) 6768401

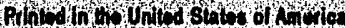

Discturacte (10.1) 


\title{
Groundwater Maps of the Hanford Site, June 1992
}

\author{
G. L. Kasza \\ M. J. Hartman \\ F. N. Hodges \\ D. C. Weekes
}

Date Published

December 1992

Prepared for the U.S. Department of Energy Office of Environmental Restoration and Waste Management

\footnotetext{
(2) Westinghouse P.O. Box 1970

Hanford Company Richland, Washington 99352

Hanford Operations and Engineering Contractor for the

U.S. Department of Energy under Contract DE-AC06-87RL10930
} 
WHC-EP-0394-5

\section{CONTENTS}

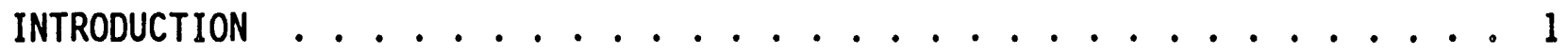

HANFORD SITE MAPS ..................... 1

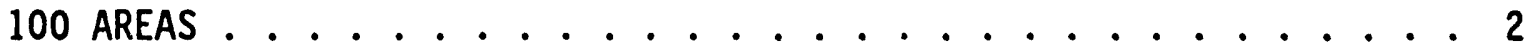
200 AREAS . . . . . . . . . . . . . . . 2

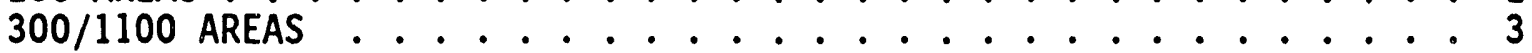

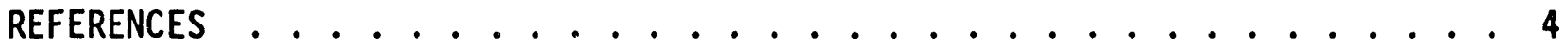

\section{FIGURES}

1 Hanford Site Location Map .................. . 5

2 Hanford Site Index Map June $1992 \ldots . . . . . . . . .66$

3 Hanford Site Depth-to-Water Map June 1992 . . . . . . . . . . . . 7

4 Hanford Site Water Table Map June 1992 ... . . . . . . . . . . 8

5100 Areas Index Map June $1992 \ldots . . . . . . . . . . .99$

6100 Areas Depth-to-Water Map June 1992 . . . . . . . . . . . . 10

7100 Areas Water Table Map June 1992 ............... 11

8200 Areas Index Map June $1992 \ldots \ldots$

9200 Areas Depth-to-Water Map June $1992 \ldots . . . . . . . . . .13$

10200 Areas Water Table Elevatiun June $1992 \ldots \ldots 14$

$11300 / 1100$ Area Index Map June $1992 \ldots \ldots 15$

$12300 / 1100$ Area Depth-to-Water Map June $1992 \ldots \ldots 16$

$13300 / 1100$ Area Water Table Map June 1992 . . . . . . . . . . . . . . 17

APPENDIX A - WATER LEVEL MEASUREMENT DATA . . . . . . . . . . A-1 
WHC-EP-0394-5

\section{INTRODUCTION}

The Groundwater Maps of the Hanford Site, June 1992 is an update to the series of reports that document the configuration of the water table in the unconsolidated sediments beneath the Hanford Site (Figure 1). Water level measurements for these reports are collected from site groundwater monitoring wells each June and December. The groundwater data are portrayed on a series of maps to illustrate the hydrologic conditions at the Hanford Site and are also tabulated in an appendix. The purpose of this report series is to document the changes in the groundwater level at Hanford as the site transitions from a nuclear material production role to environmental restoration and remediation. In addition, these reports provide water level data in support of the site characterization and groundwater monitoring programs on the Hanford Site. Groundwater maps of the Hanford Site are prepared for the U.S. Department of Energy, Office of Environmental Restoration and Waste Management, by the Hanford Site Operations and Engineering Contractor, Westinghouse Hanford Company (WHC).

This report presents the June 1992 water level data for the uppermost aquifer beneath the Hanford Site. During that month, the depth to the groundwater was measured in over 700 wells by personnel from WHC Environmental Field Services Group. The procedure for measuring the depth to water and recording the data is contained in Environmental Investigations and Site Characterization Manual (EII 10.2) Measurement of Groundwater Levels

(WHC 1988). This report and the groundwater maps were prepared by staff members of the WHC Geosciences Group, Environmental Division. Vater level data from December 1991 were reported in Kasza et al. (1992).

Appendix A lists the well identification number, depth to water, casing elevation and the water level elevation for each well measured during June 1992. An explanation of the data is included along with the well index map, the depth to water map, and the contoured map of the water table surface for the entire site and for the specific operational areas on the site. For clarity, the locating prefixes have been omitted from all well numbers shown on the maps. Wells in the 100 Areas have the prefix 199; wells in the 200 Areas have the prefix 299; wells in the 300 Area have a 399 prefix; those in the 1100 Area have a 1199 prefix and the wells outside these areas have the prefix 699. An explanation of the Hanford well numbering system and a listing of the survey coordinates for each well drilled prior to 1989 is ?isted in McGhan (1989).

\section{HANFORD SITE MAPS}

The Hanford Site maps depict the configuration of the surface of the unconfined aquifer beneath the majority of the Hanford Site, which is located south and west of the Columbia River. The site maps are: Index Map of the Hanford Site (Figure 2); Site Depth-to-Water Map (Figure 3); and Hanford Site Water Table Map (Figure 4). The site water table map is a composite of the detailed maps of the operating areas and data from the groundwater monitoring 
wells located outside the operating areas. The borders of the operating areas maps are represented accurately on the site maps for ease in comparing the maps.

\section{AREAS MAPS}

For the purposes of this report, the 100 Areas comprises the various 100 Area reactor facilities and the surrounding land south of the Columbia River and north of Gable Mountain and Gable Butte. Reactor operations have ceased in all of the facilities, and environmental restoration activities are in progress. Maps for this area include: Index Map of the 100 Areas Groundwater Monitoring Wells (Figure 5); 100 Areas Depth-to-Water Map (Figure 6); and 100 Areas Water Table Map (Figure 7). The 100 Areas maps are based on June 1992 field measurements from those wells that are located north of Hanford Site coordinate $N 56,000$ and completed in the unconsolidated sediments. Wells used are completed within $100 \mathrm{ft}$ of the average water table since vertical gradients are not significant on the scale of the map.

Data were averaged for wells with multiple measurements in June. A representative set of wells from the reactor areas were chosen where there are heavy concentrations of wells (e.g., 100-N and 100-H areas). Hydrographs were plotted for wells with anomalous head values. If the measurement for June was an outlier, the data point was not included in constructing the maps.

Surveyed locations of wells generally are as listed in Hanford Wells (McGhan 1989). However, several wells were located in the document using the local 100-N grid system. Wells drilled later than 1989 are not included in the document. Hartman (1992) lists the 100-N area wells converted to the Hanford Plant coordinate system. The new 100-D wells were surveyed to the Hanford Plant coordinates.

Well casing elevations generally are as listed in McGhan (1989). Recently installed wells in the 100-D and 100-N areas were surveyed to the local 100-D and 100-N vertical datums and also to the National Vertical Geodetic Datum of 1929. The local datums are presumed to correspond to McGhan (1989) and were used in the maps presented in this document.

The $N 65,000$ match line is included on the maps of the 100 and 200 areas for convenience.

\section{AREAS MAPS}

These maps encompass the 200 East Area and 200 West Area and the surrounding vicinity on the Hanford Site that was once referred to as the Separations Area. The semiannual measurement of water levels in the 200 Areas was performed on the more than 180 selected wells that comprise the Operational Groundwater Monitoring Network (Serkowski 1989). The Operational Groundwater Monitoring Network provides water level measurements for the determination of the water table configuration and for the water quality sampling of the groundwater beneath and surrounding the active and inactive chemical processing and waste management facilities in the 200 Areas. Water level data from additional non-Network wells are included on the maps to 
provide supplemental information for a more complete interpretation. The wells selected for use were reviewed to assure that they were completed in the unconsolidated sediments and screened or perforated in the upper part of the unconfined aquifer.

The 200 Areas set of maps consists of: 200 Areas Monitoring Well Index Map (Figure 8), 200 Areas Depth-to-Water Map (Figure 9), and 200 Areas Water Table Map (Figure 10). These maps are based on June 1992 water level measurements for the unconfined aquifer. Representative wells were selected to portray the water level data on the maps where the concentration of monitoring wells would produce a crowded illustration.

\section{$300 / 1100$ AREAS MAPS}

This section contains June 1992 water table measurements for the 300 Area and the adjacent 1100 Area and Horn Rapids Landfill. Wells used for obtaining the measurements were screened or perforated in the upper part of the unconfined aquifer except in a few cases where the top of the screen was slightly below the top of the unconfined aquifer. Water table data from new monitoring wells with pressure transducers were added to the data set to provide a more accurate water table map. Wells having pressure transducers include (prefixed by 699-), S29-E16A, S19-E14, S22-E9A, S27-E9A, and S28-E12. A pressure transducer is also installed in SWS-1 for monitoring the Columbia River stage at the 300 Area. Readings from the pressure transducers are taken every hour and stored in a data logger that is then downloaded to a computer for calculation of water level elevations. The water level measurement that corresponded with the time that the nearby wells without transducers were manually measured was used since the water level in the unconfined aquifer near the Columbia River responds rapidly to variations in the river stage.

The major influences on water table elevations in the map area are river level fluctuations, water discharge to the 300 Area Process Trenches and sanitary system, and river water recharged into the city of Richland well field near the 1100 Area. Water table fluctuations depend on recharge from the Yakima River to the west. The top of the unconfined aquifer is very close to the Hanford formation/Ringold Formation contact. Generally, west of an imaginary north-south line near the west boundary of the 300 Area the unconfined aquifer is within the Ringold Formation. East of the line it is within the Hanford formation.

The data for this area are presented as the 300/1100 Area Index Map (Figure 11), the 300/1100 Area Depth-to-Water Map (Figure 12), and a 300/1100 Area Water Table Map (Figure 13). National Geodetic Vertical Datum of 1929 is used as the vertical datum for the maps and Hanford Plant coordinates used for horizontal position of the wells. Both datums are in feet. 


\section{REFERENCES}

Hartman, M. J., 1992, Borehole Completion Package for Wells 199-N-71, $199-N-72,199-N-73$ and 199-N-74, WHC-SD-EN-DP-040, Westinghouse Hanford Company, Richland, Washington.

Kasza, G. L., M. J. Hartman, F. N. Hodges, and D. C. Weekes, 1992 , Ground Water Maps of the Hanford Site--December 1991, WHC-EP-0394-4, Westinghouse Hanford Company, Richland, Washington.

McGhan, V. L., 1989, Hanford Wel7s, PNL-6907, Pacific Northwest Laboratory, Richland, Washington.

Serkowski, J. A. and W. A. Jordan, 1989, Operational Groundwater Monitoring at the Hanford Site--1988, WHC-EP-0260, Westinghouse Hanford Company, Richland, Washington.

WHC, 1988, Environmental Investigations and Site Characterization Manual, WHC-CM-7-7, Westinghouse Hanford Company, Richland Washington. 
WHC-EP-394-5

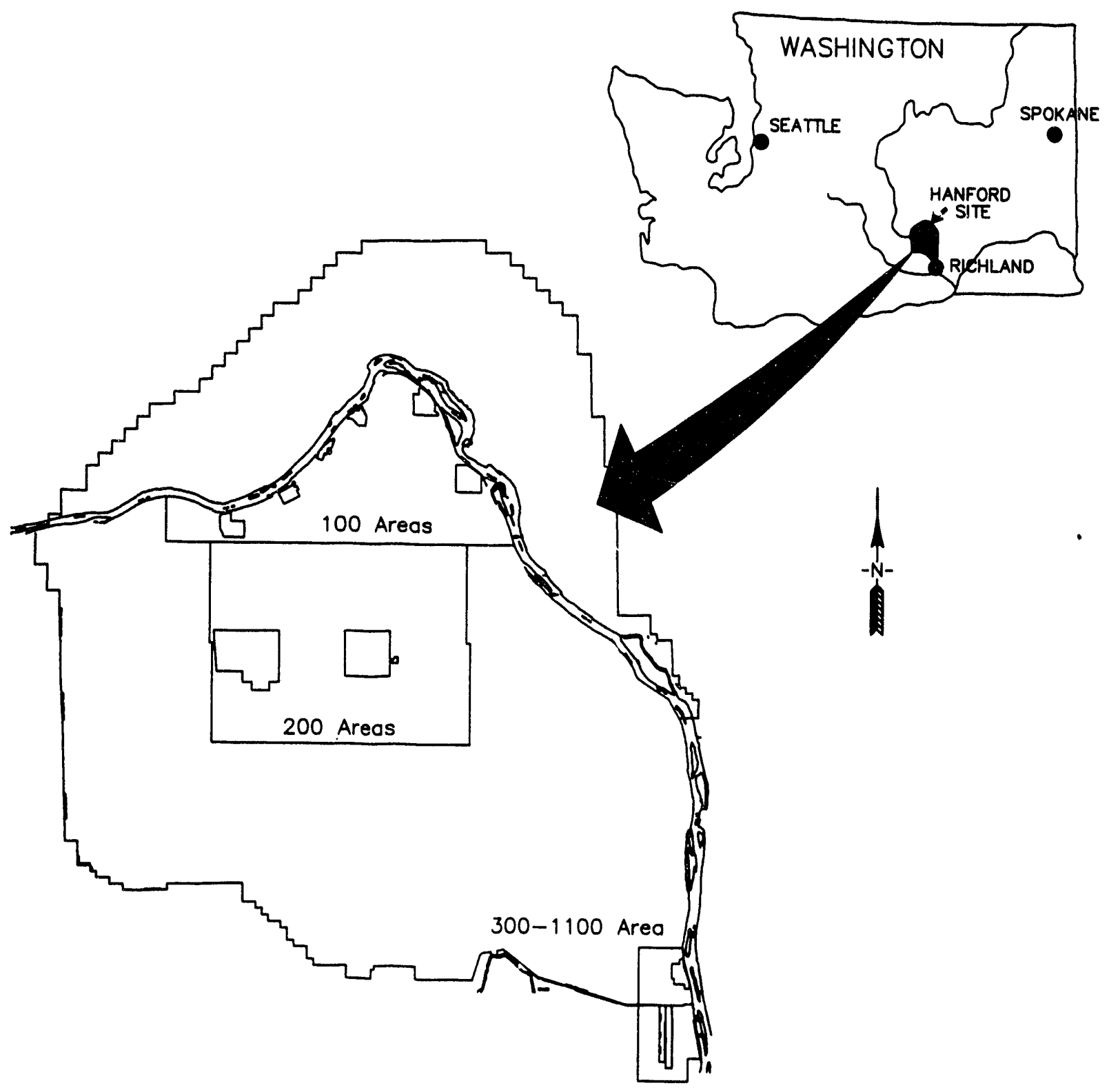

Figure 1. Hanford Site Location Map. 


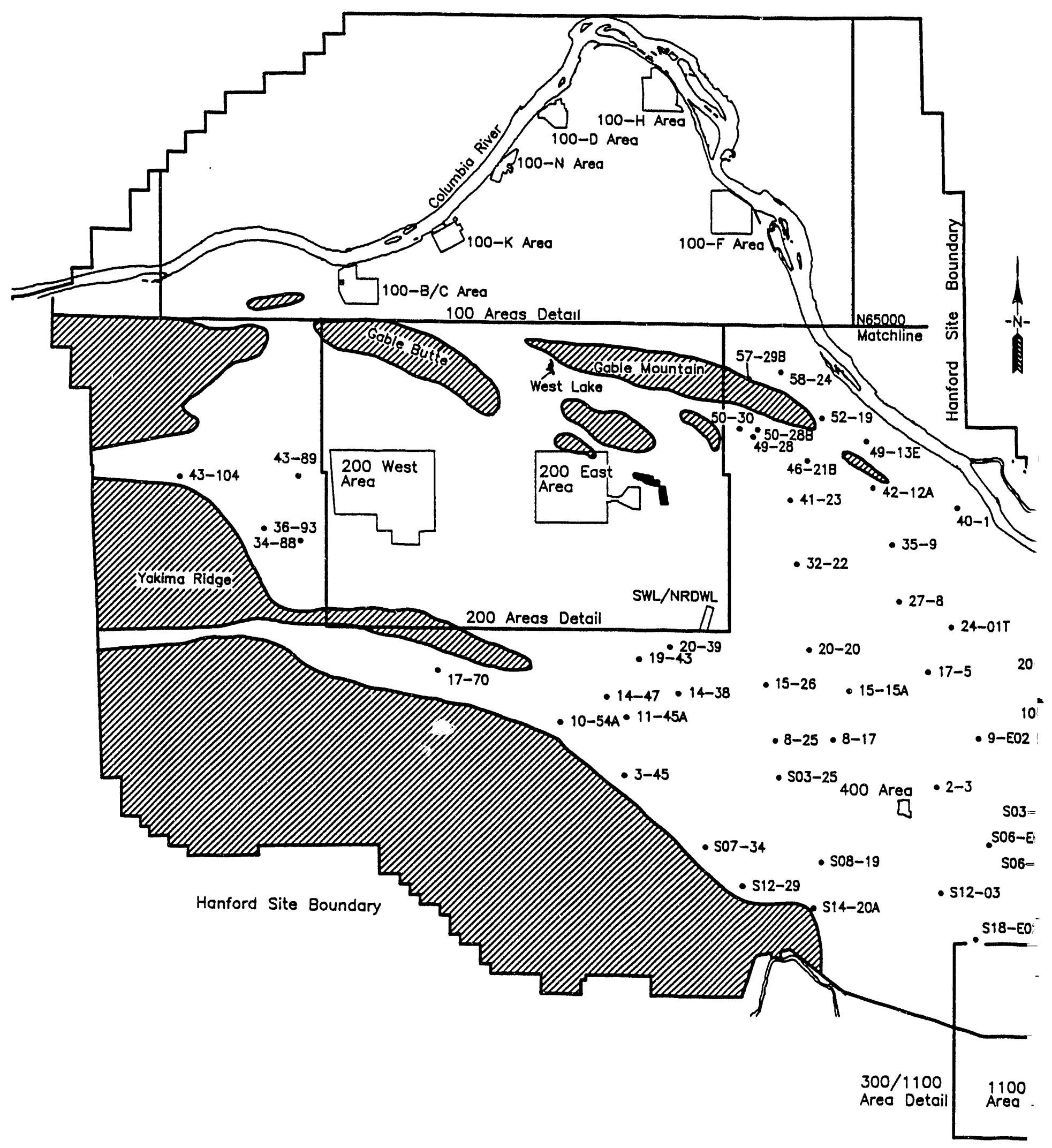




\title{
Hanford Site Index Map June 1992
}

\author{
32-22 Identification of monitoring well used \\ to prepare this map. All wells on this \\ map are prefixed by 699-. \\ Ponds \\ Areas where the basalt surface is \\ generally above the water table \\ The Hanford site index map has been \\ prepared by the Geosciences Group, Environmental \\ Division, of Westinghouse Hanford Company.
}

Note: To convert to metric, multiply elevation ( $\mathrm{ft}$ ) by 0.3048 to obtain elevation $(\mathrm{m})$.

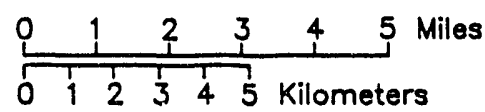

Figure 2 


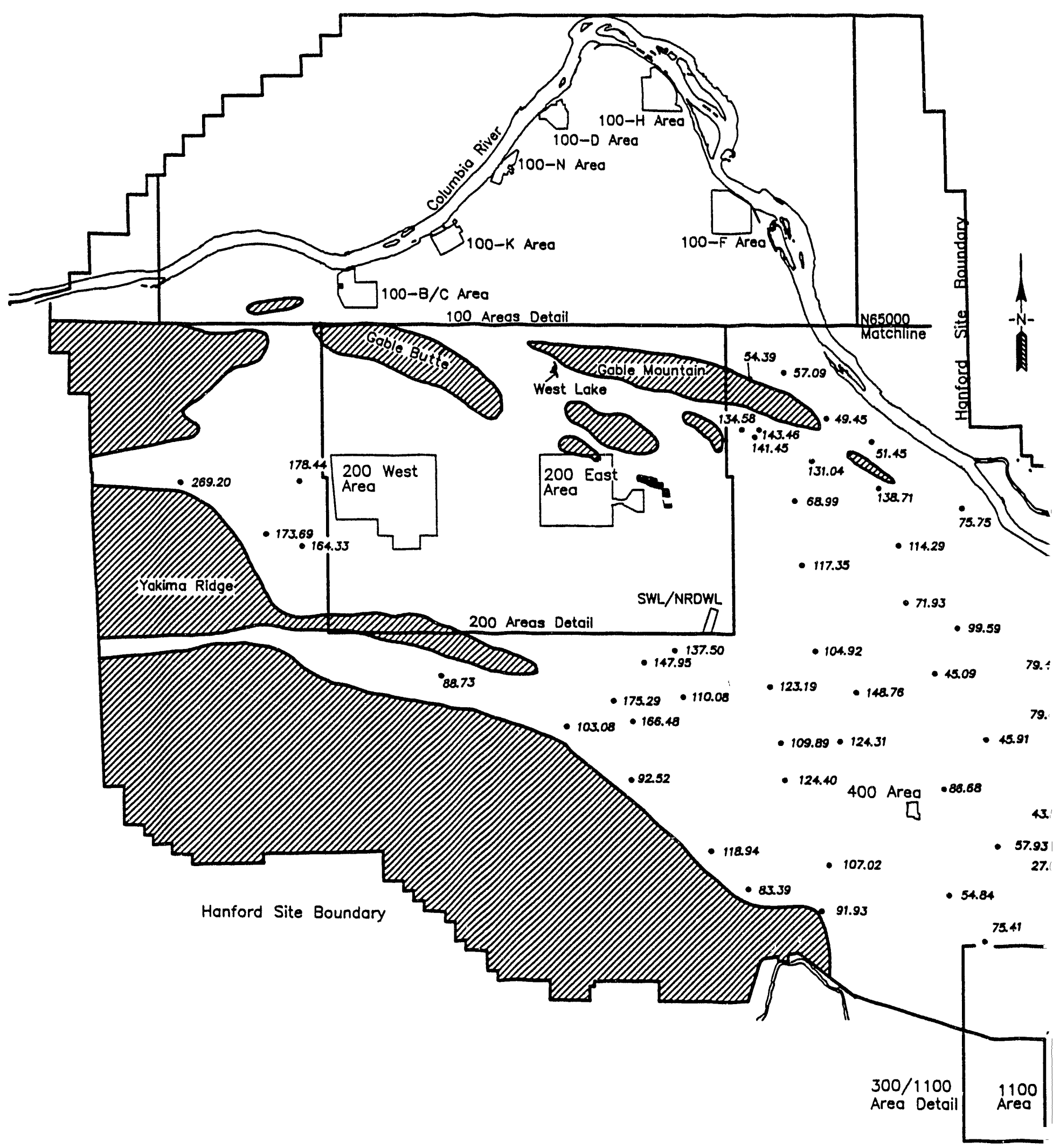




\title{
Hanford Site Depth-to-Water Map June 1992
}

\author{
36.14 Depth to water in feet, as \\ measured from well reference \\ mark (generally top of casing) \\ to ground water surface \\ Ponds \\ QIII Areas where the basalt surface is \\ generally above the water table \\ The Hanford Site depth-to-water map has been \\ prepared by the Geosciences Group. Environmental \\ Division, of Westinghouse Hanford Company.
}

Note: To convert to metric, multiply elevation (ft) by 0.3048 to obtain elevation $(\mathrm{m})$.

\begin{tabular}{lllllll}
0 & 1 & 2 & 3 & 4 & 5 \\
\hline & & 1 & 1 & 1 & 1 & 1 \\
0 & 1 & 2 & 3 & 4 & 5 & Kilometers
\end{tabular}




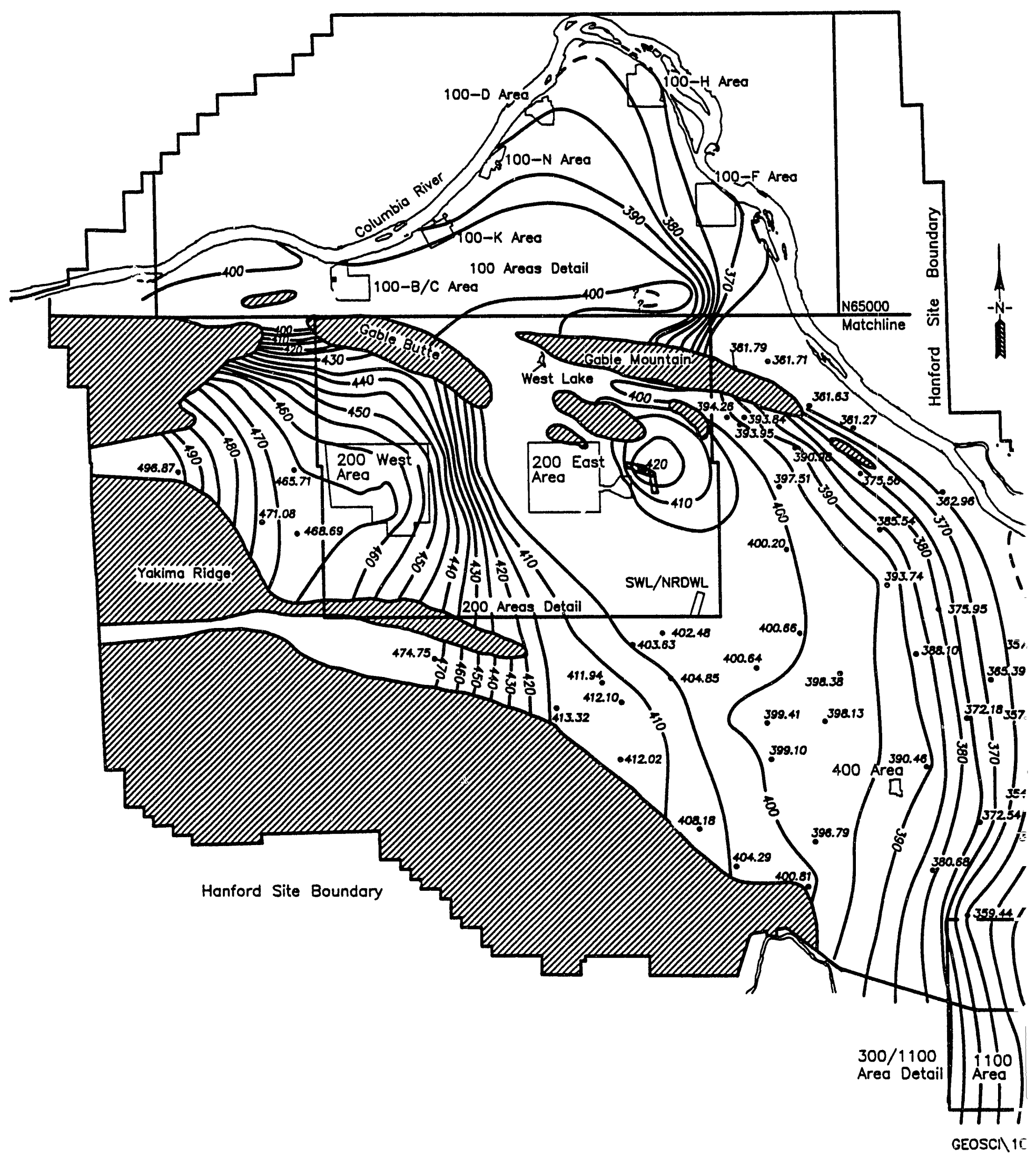


WHC-EP-0394-5

\title{
Hanford Site Water Table Map June 1992
}

\author{
411.49 Water table elevation (feet \\ above mean sea level) \\ Water table contours in feet \\ above mean sea level \\ Contour Interval $=5 \mathrm{ft}$ \\ Contour Lines are dashed \\ where approximated. \\ Ponds \\ Areas where the basalt surface is \\ generally above the water table \\ The Hanford Site water table map has been \\ prepared by the Geosciences Group. Environmental \\ Division, of Westinghouse Hanford Company.
}

Note: To convert to metric, multiply elevation (ft) by 0.3048 to obtain elevation (m).

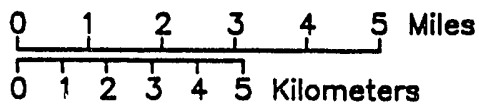




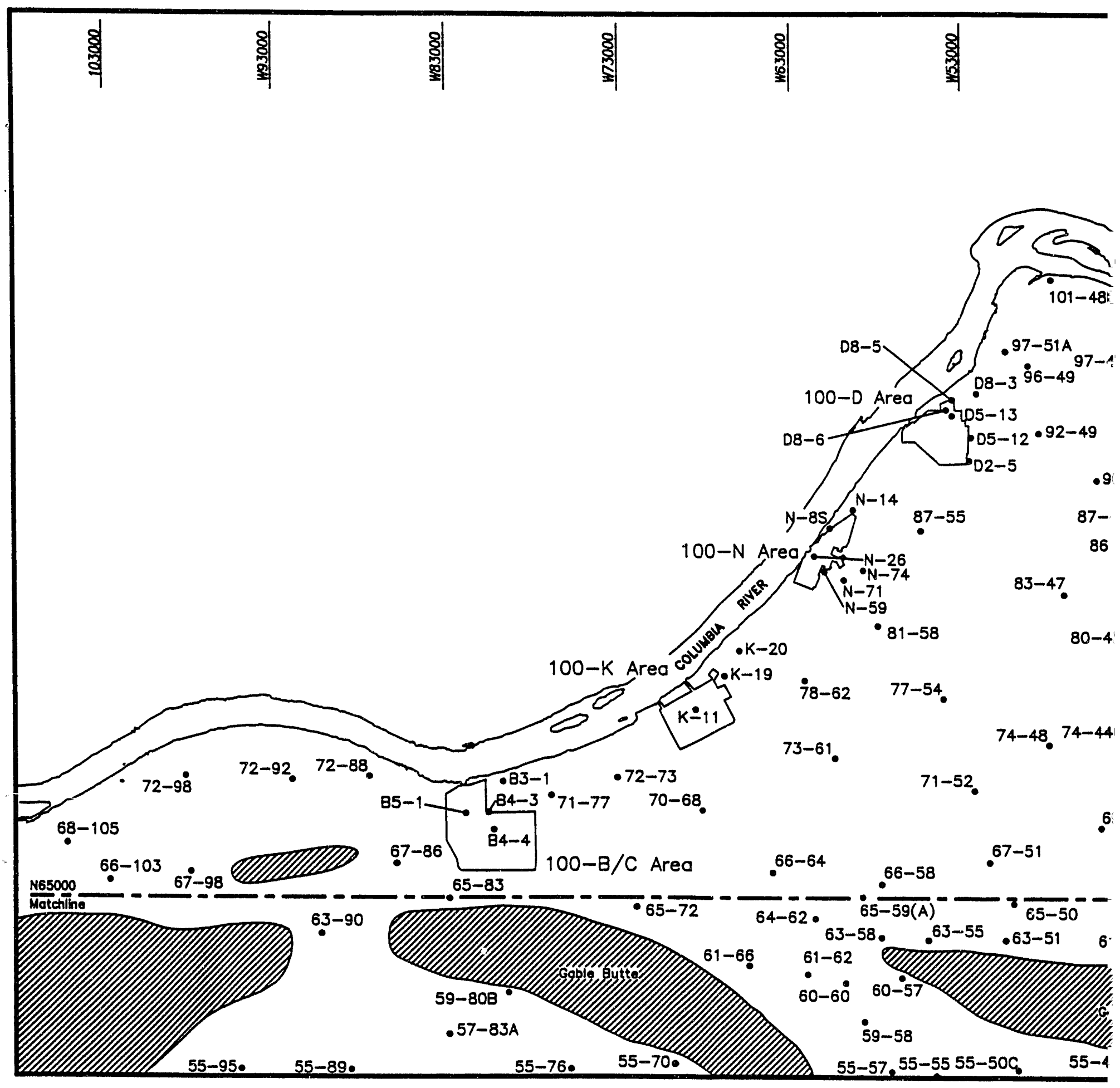




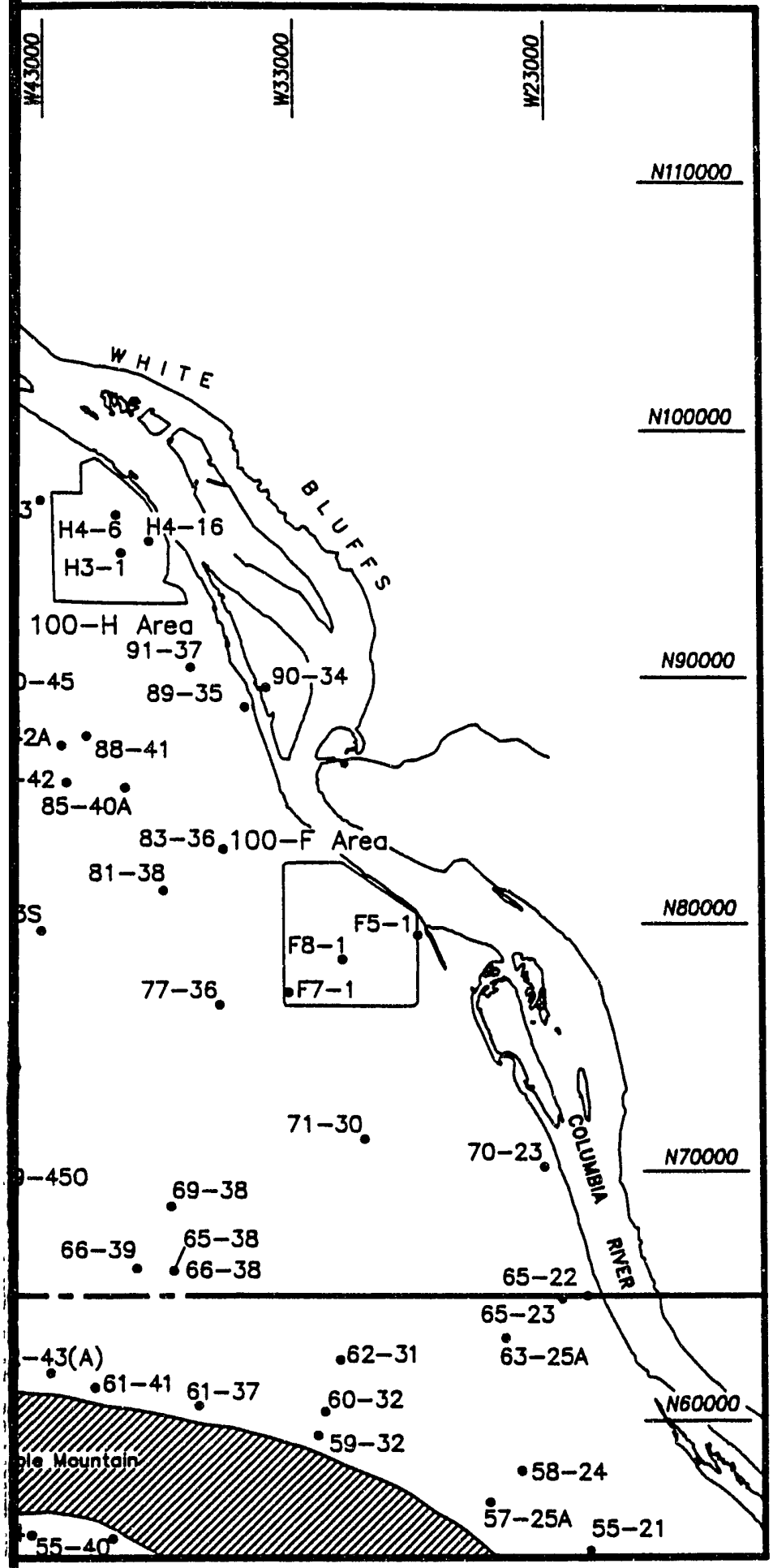

GEOSCN 091892-A

\section{Areas Index Map June 1992}

77-40 Identification of Groundwater Monitoring Well Used to Prepare Map

Areas where the basalt surface is generally above the water table

The 100 Areas Index Map has been prepared by the Geosciences Group, Environmental Division, of Westinghouse Hanford Company.

Note: To convert to metric, multiply elevation (ft) by 0.3048 to obtain elevation (m).

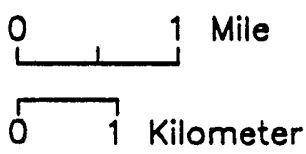




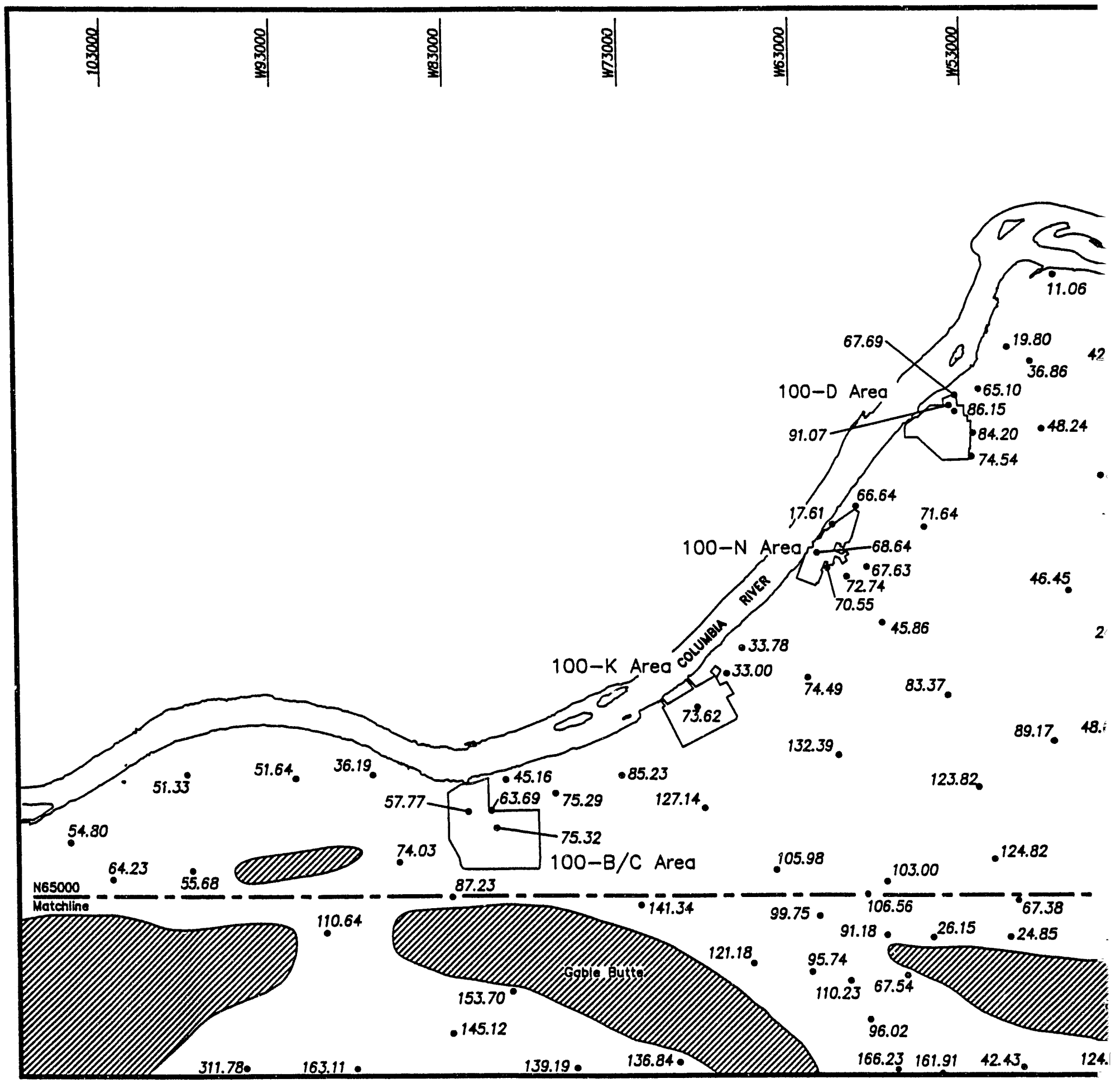




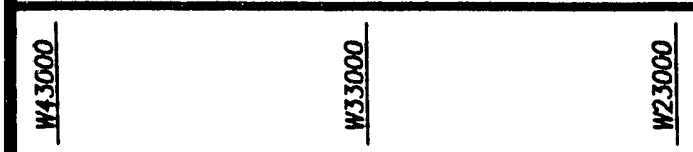

N110000

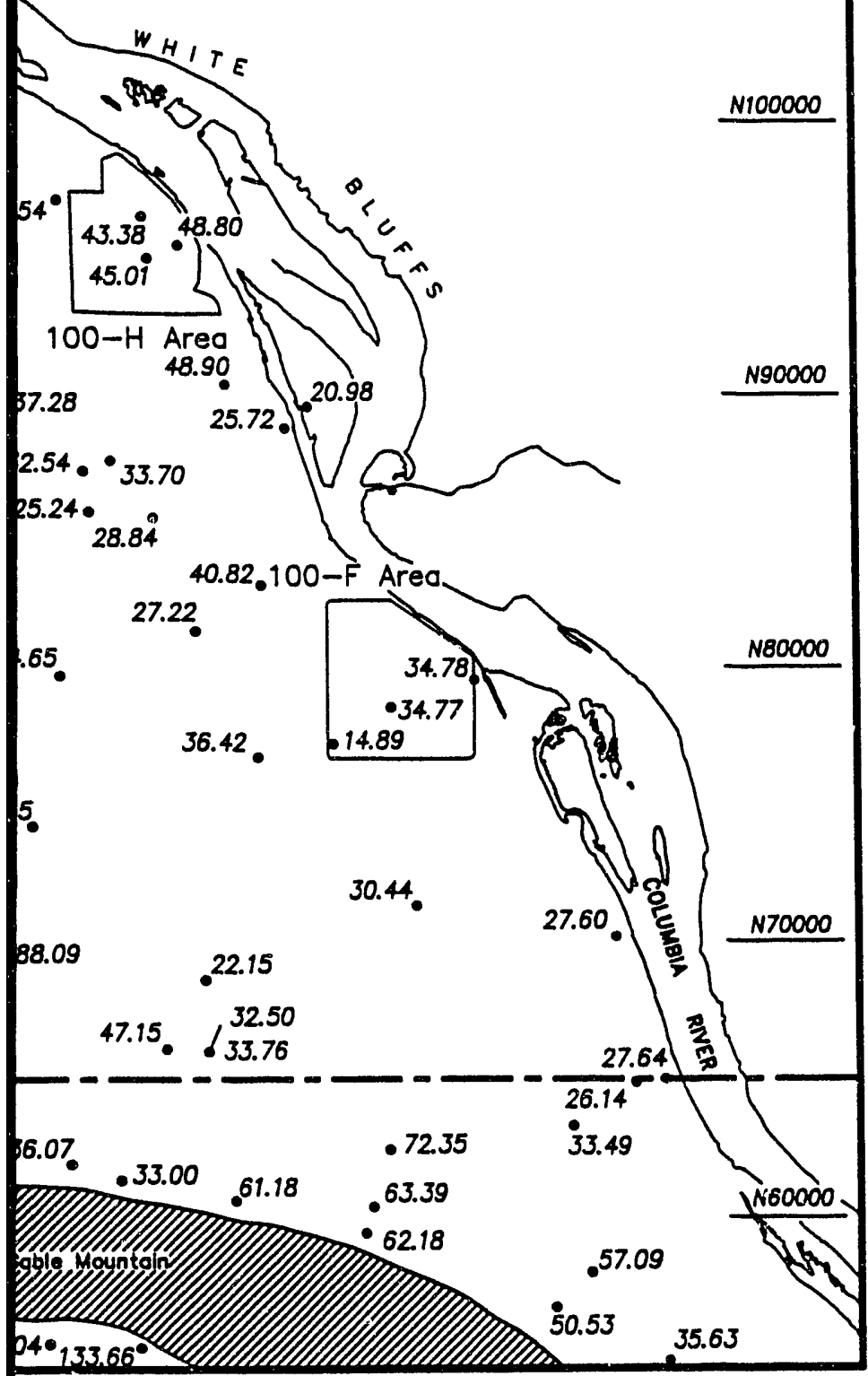

GEOSCN $091892-8$

\section{Areas \\ Depth-To-Water Map June 1992}

36.14 Depth to water, as measured from well reference mark (generally top of casing) to groundwater surface.

UIII) Areas where the basalt surface is generally above the water table

The 100 Areas depth-to-water map has been prepared by the Geosciences Group, Environmental Division, of Westinghouse Hanford Company.

Note: To convert to metric, multiply elevation ( $\mathrm{ft}$ ) by 0.3048 to obtain elevation (m).

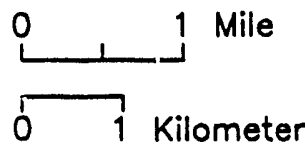

Figure 6 


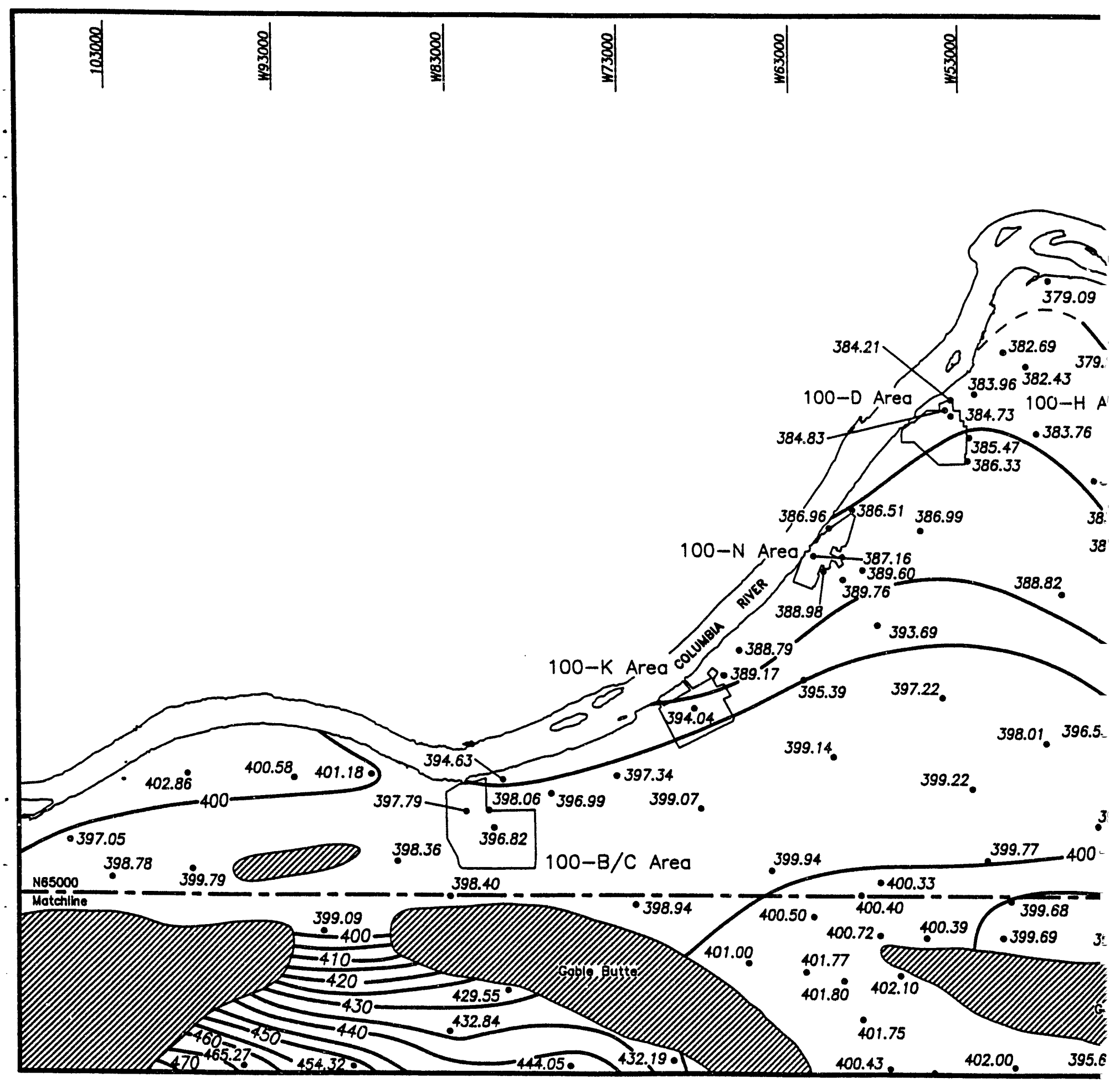




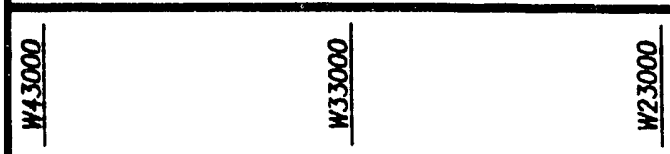

$N 110000$

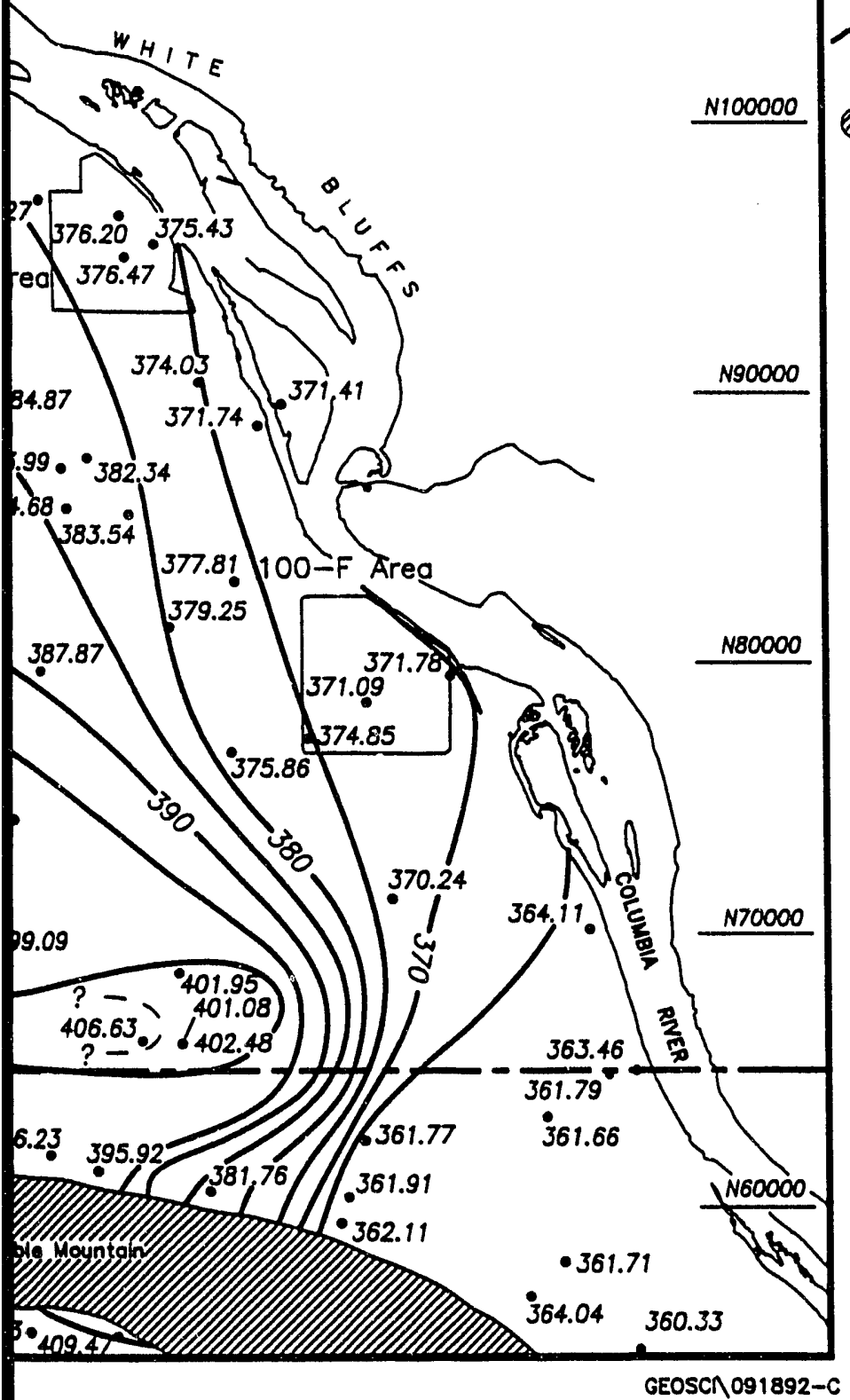

\section{Areas Water Table Map June 1992}

388.60 Water table elevation,

- (feet above mean sea level)

$400-$ Groundwoter table elevation Contour Interval $=5 \mathrm{ft}$

UII Areas where the bosalt surface is generally above the water table

The 100 Areas water table map has been prepared by the Geosciences Group, Environmental Division, of Westinghouse Hanford Company.

Note: To convert to metric, multiply elevation (ft) by 0.3048 to obtain elevation ( $m$ ).

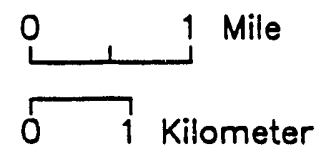

$\overbrace{-N-}^{-N}$ 


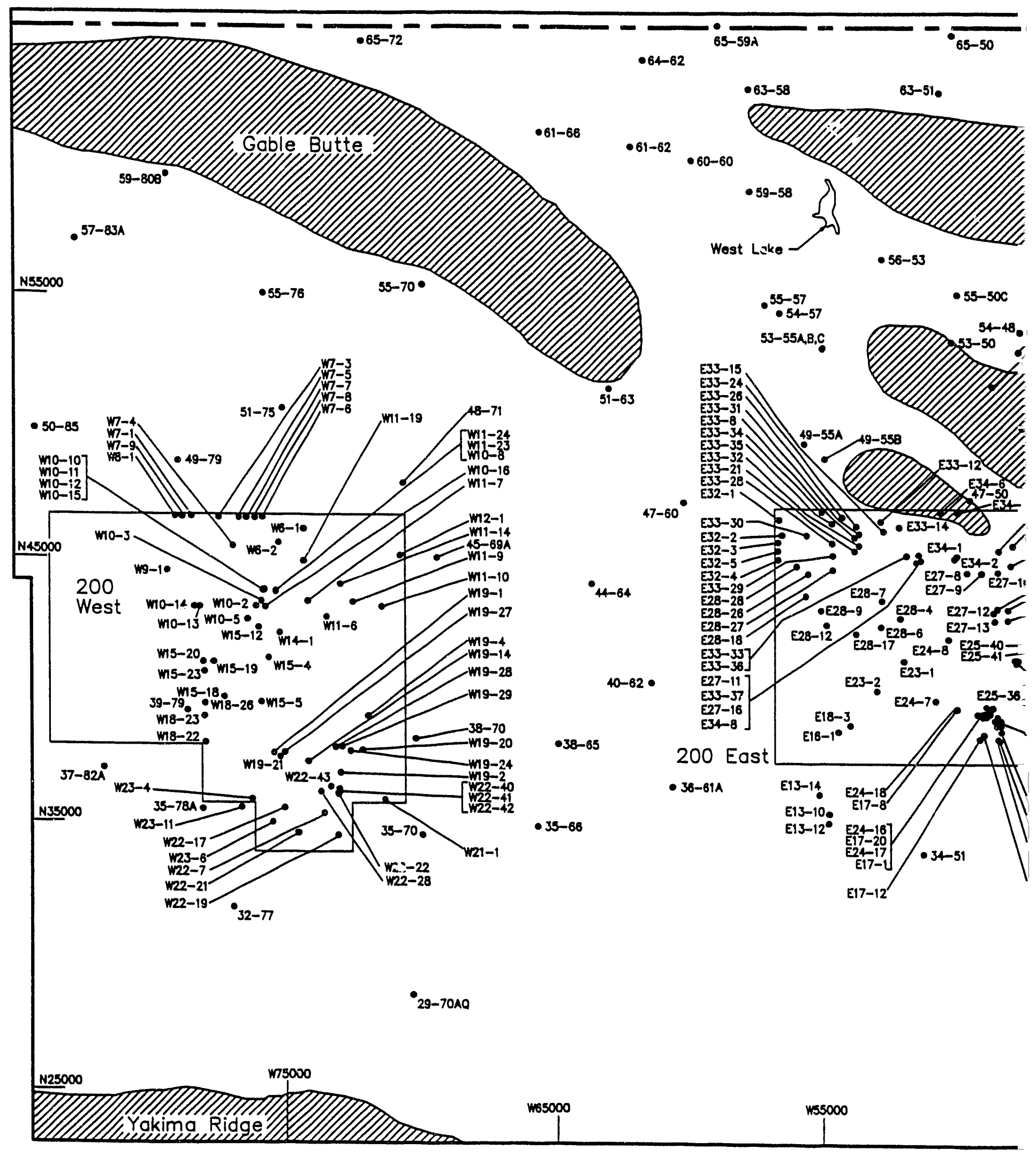




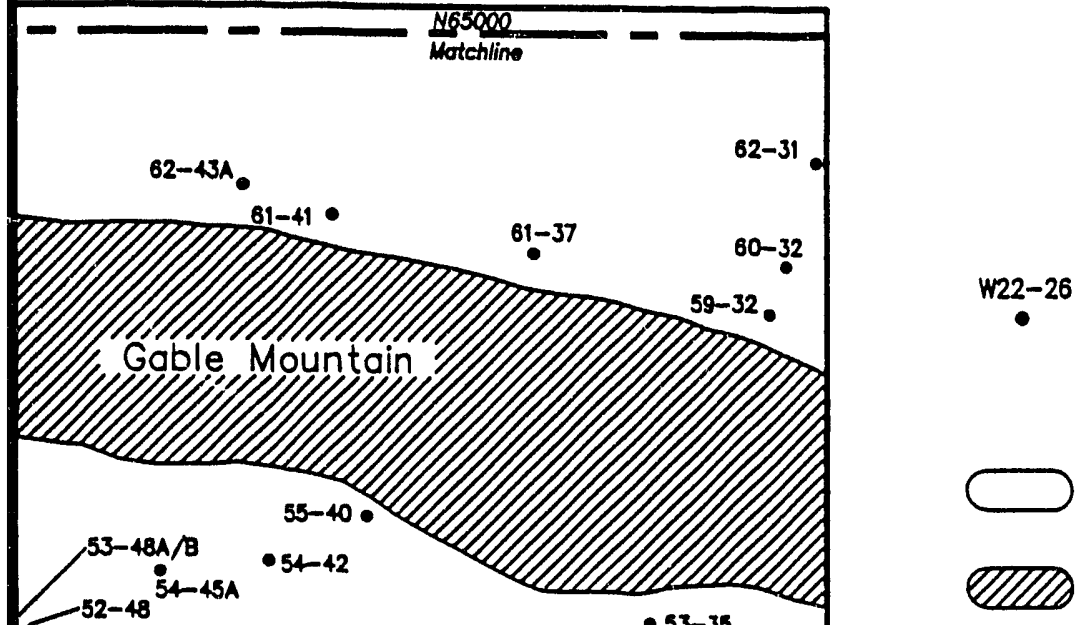

200 Areas Index Map June 1992

W22-26 Identification of monitoring well used to prepare map. Well Numbers storting with $E$ or $W$ are prefixed by 299 - and all others ore prefixed by $699-$.

Ponds

Areas where the basalt surface is generally above the water table

The 200 Areas index map has been prepared by the Geosciences Group. Environmental Division, of Westinghouse Hanford Company.

Note: To convert to metric, multiply elevution ( $\mathrm{ft}$ ) by 0.3048 to obtain elevation (m).
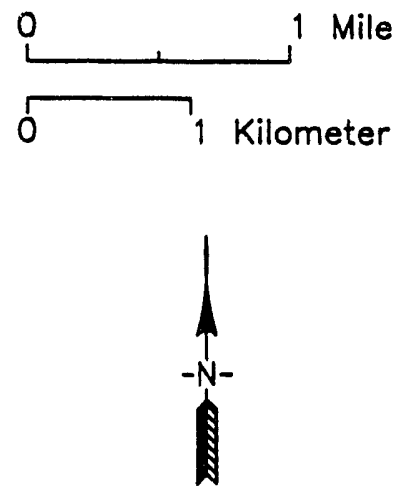

W45000

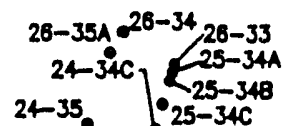

Figure 8 


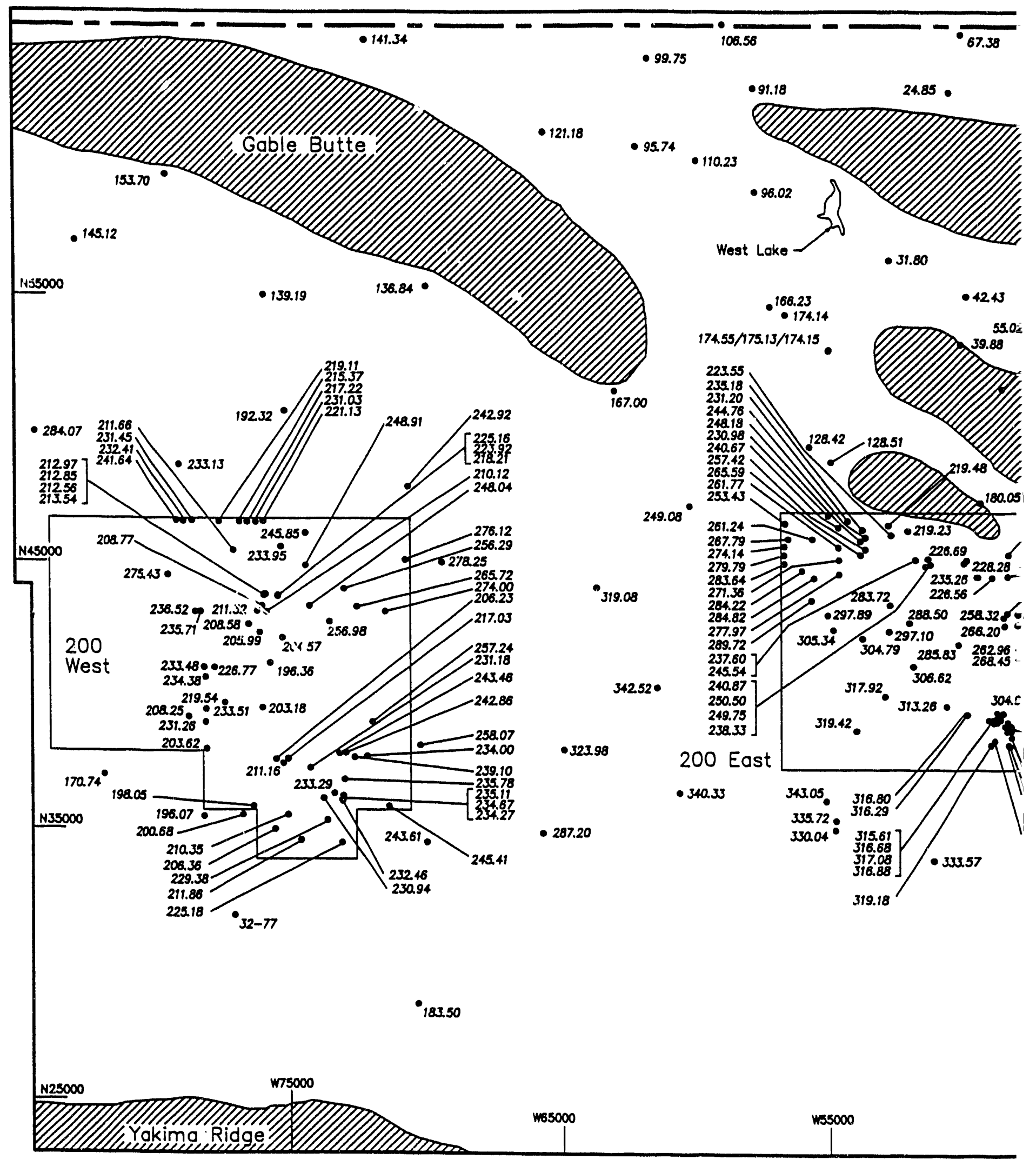




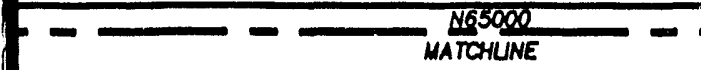

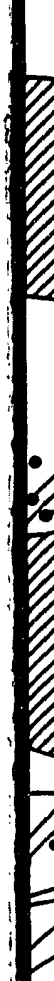
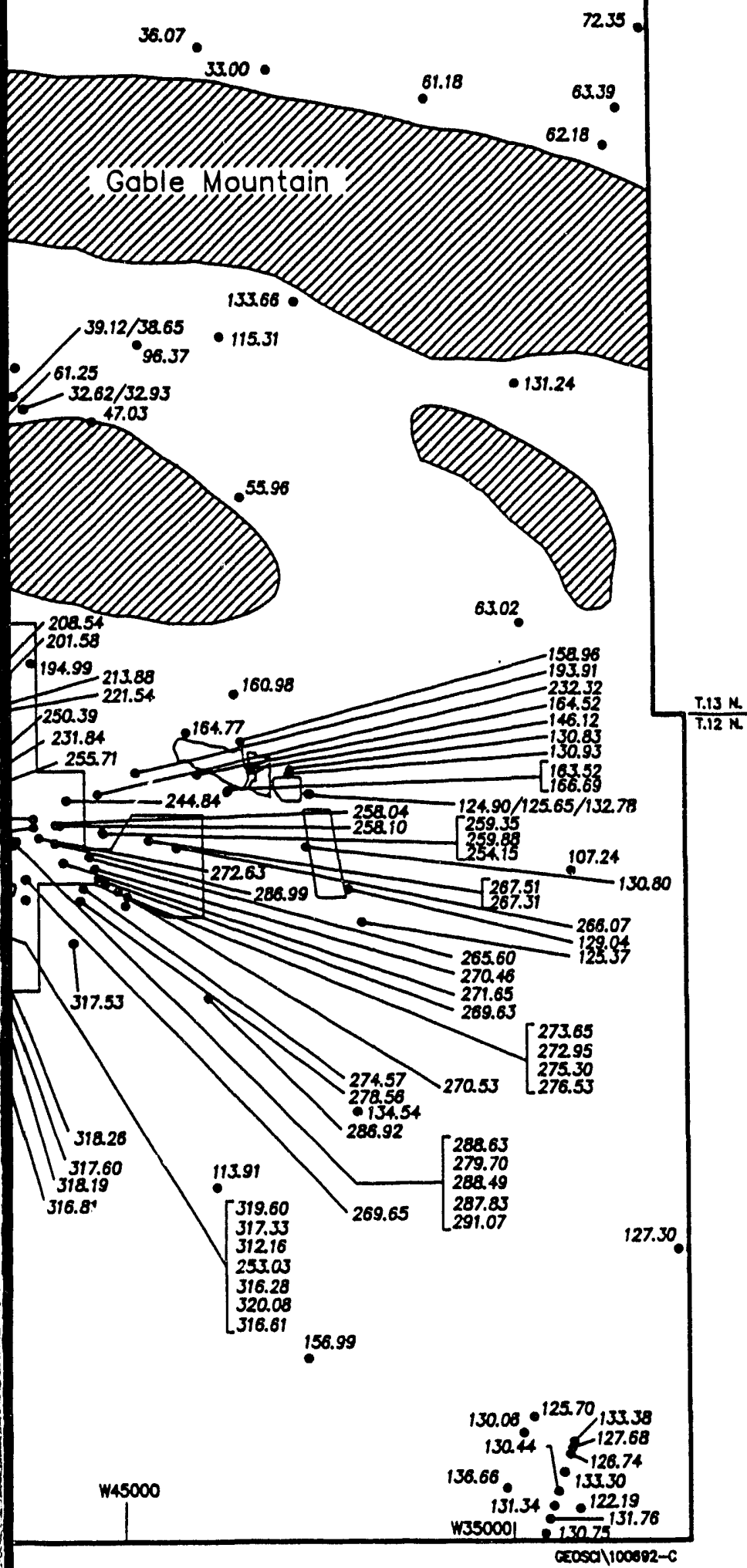

\section{Areas \\ Depth-to-Water Map June 1992}

36.14 Depth to water in feet, as measured from well reference mork (generally top of cosing) to ground water surface

\section{Ponds}

Areos where the basalt surface is generally above the woter table

The 200 Areas depth-to-water map has been prepared by the Geosciences Group, Environmental Division, of Westinghouse Hanford Company.

Note: To convert to metric, multiply elevation ( $\mathrm{ft}$ ) by 0.3048 to obtain elevation (m).

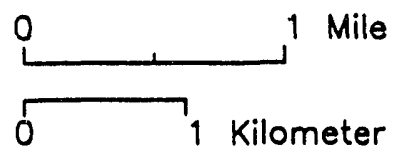

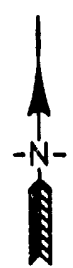




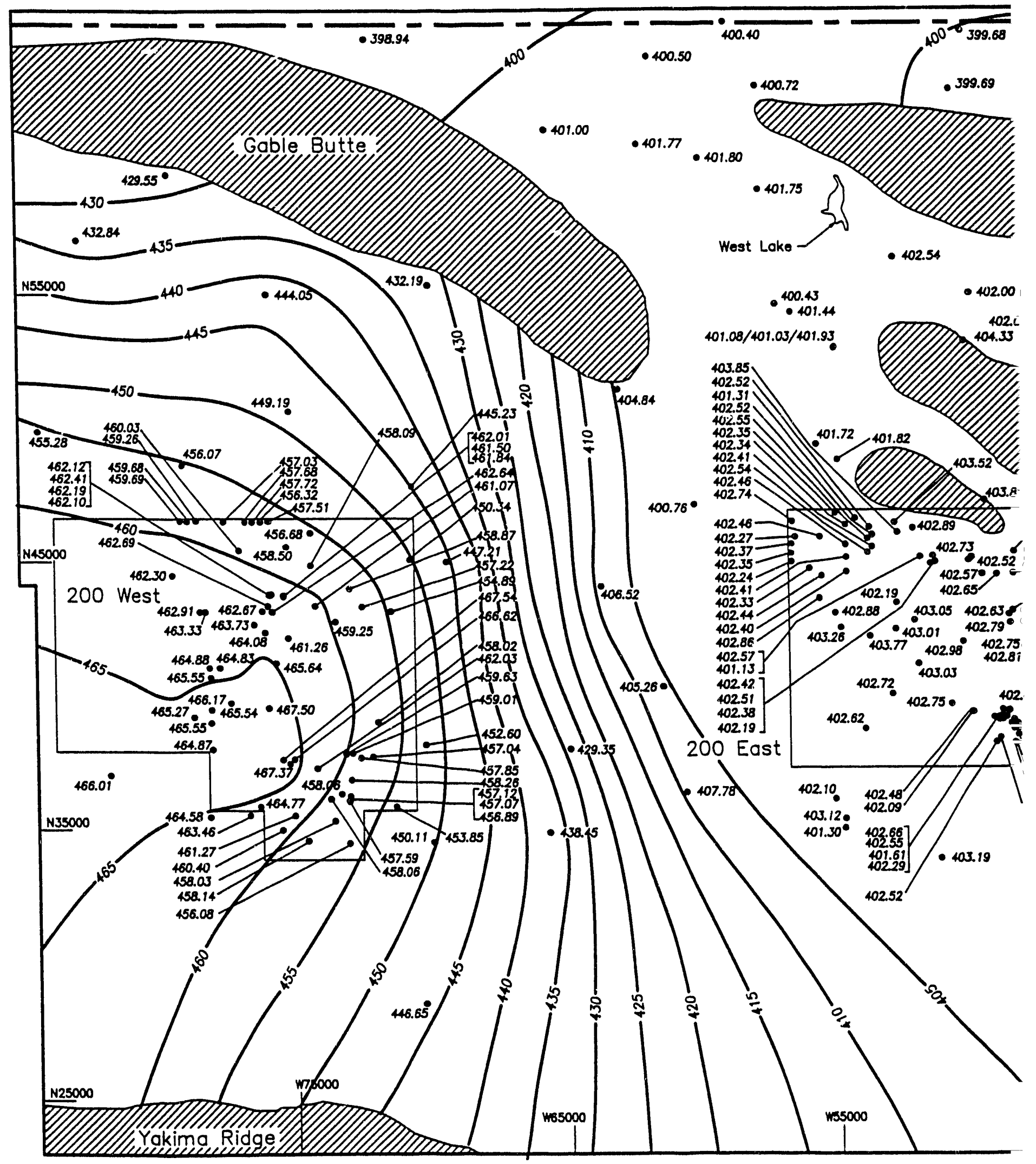




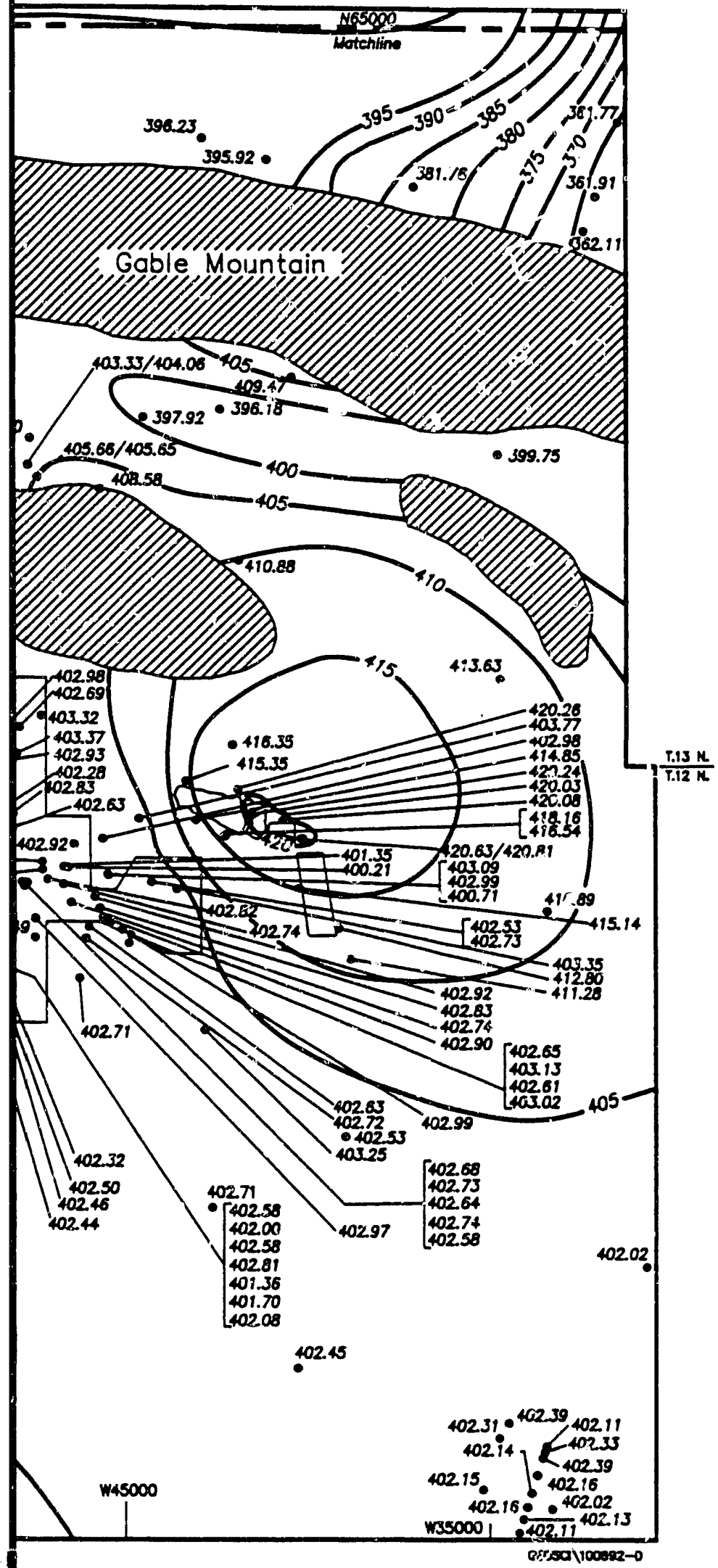

\title{
200 Areas \\ Water Table Elevation June 1992
}

\author{
411.49 Water table elevation (feet \\ above mean sea level) \\ Groundwater table elevotion \\ contour interval $=5 \mathrm{ft}$ \\ Ponds \\ Areas where the bosalt surface is \\ generally above the water table
}

The 200 Areas water toble elevation map has been prepared by the Geosciences Group. Environmental Division, of Westinghouse Hanford Company.

Note: To convert to mietric, multiply elevation (ft) by 0.3048 to obtain elevation (m).

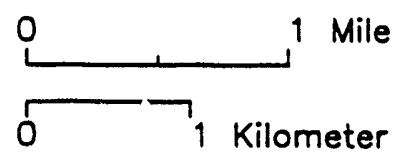

Figure 10 


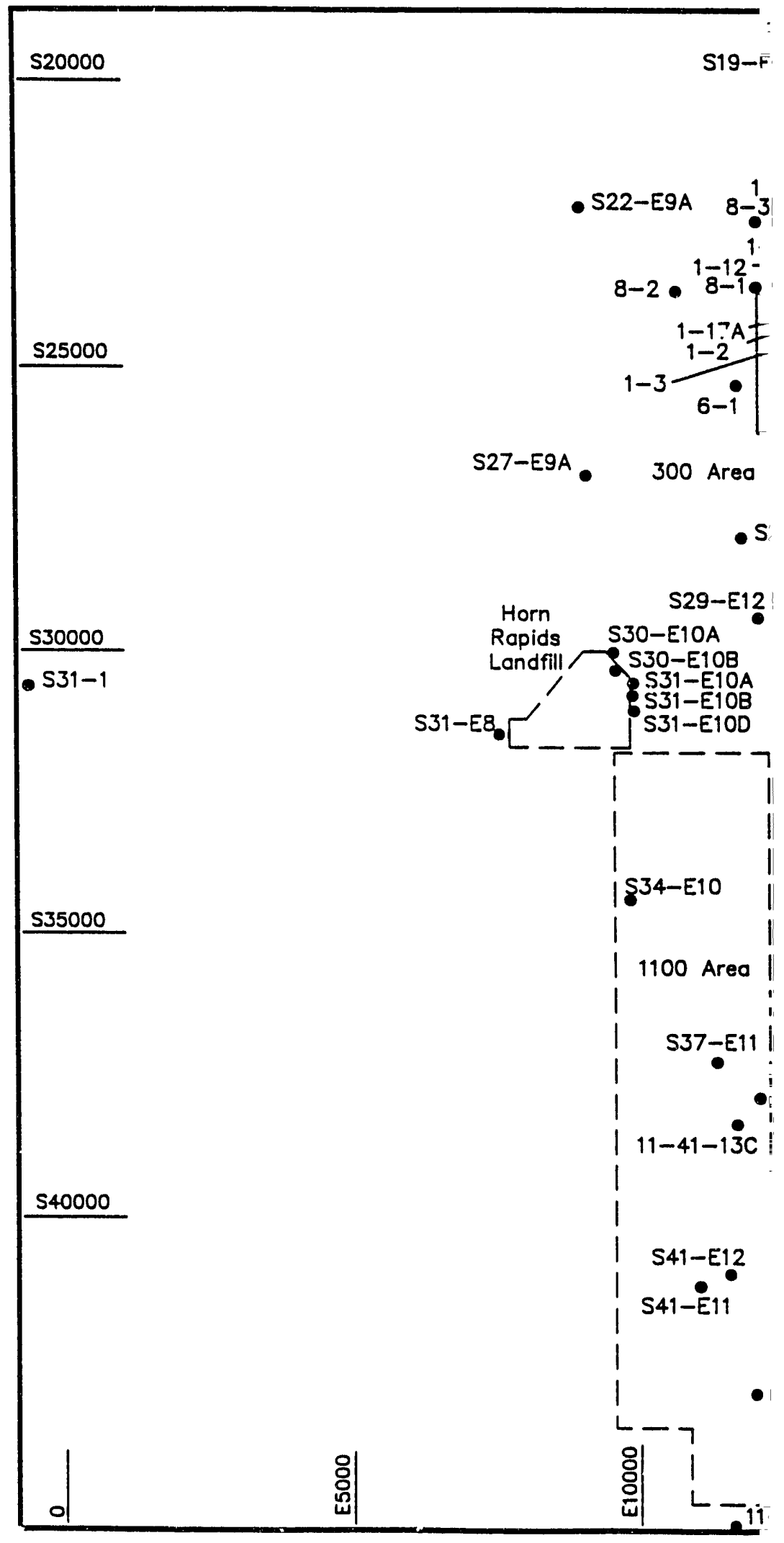


WHC-EP-0394-5

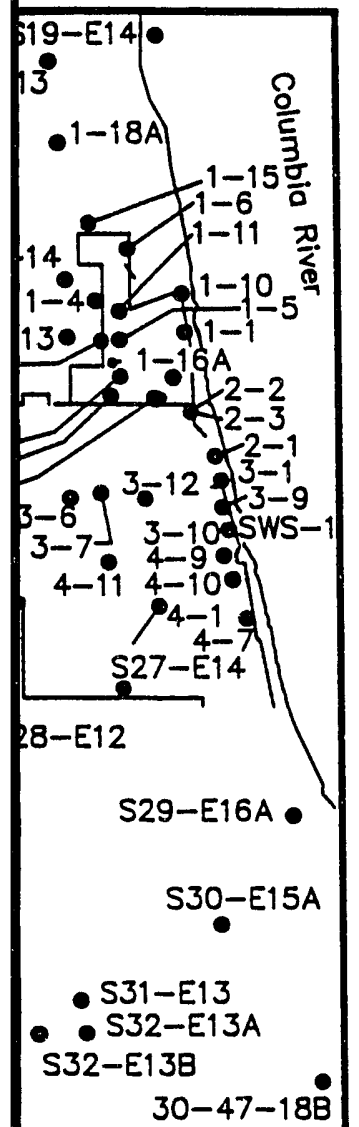

S34-E15

- S36-E13A

S36-E12B

S37-E14

$\$ 38-E 12 A$

S40-E14

- S41-E13A

$S 43-E 12$

$-34-13$

임

\section{0/1100 Area \\ Index Map June 1992}

3-1 Identification of monitoring well

- used to prepare map. Well Numbers

starting with $S$ are prefixed by

699- and well numbers starting with a single digit are prefixed

by 399-.

The 300 Areo index map has been

prepared by the Geosciences Group, Environmental

Division, of Westinghouse Hanford Company.

Note: To convert to metric, multiply

elevation (ft) by 0.3048 to obtain

elevation (m).

0 Mile
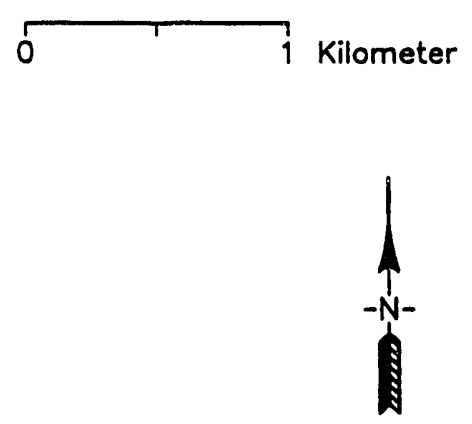

Figure 11 


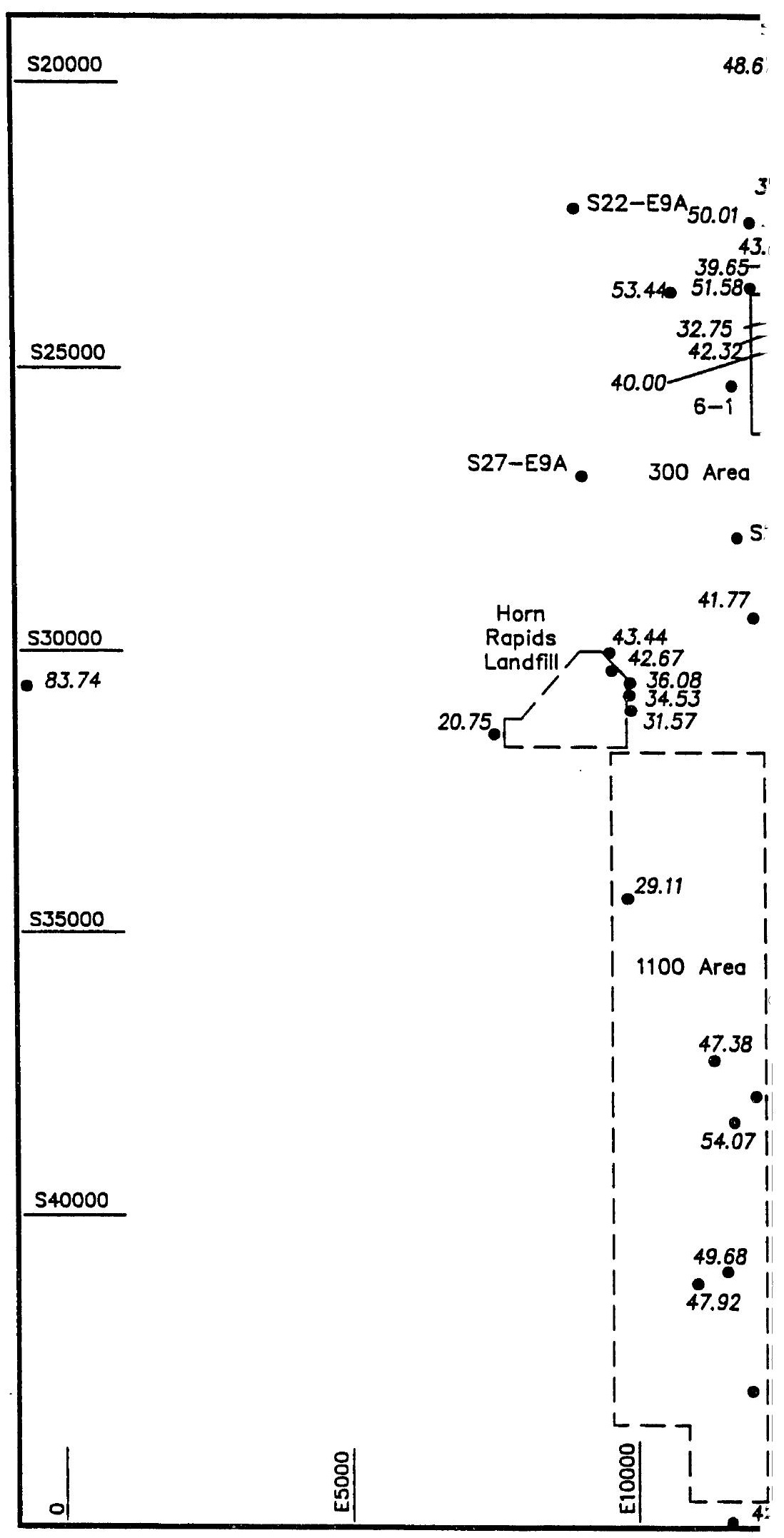




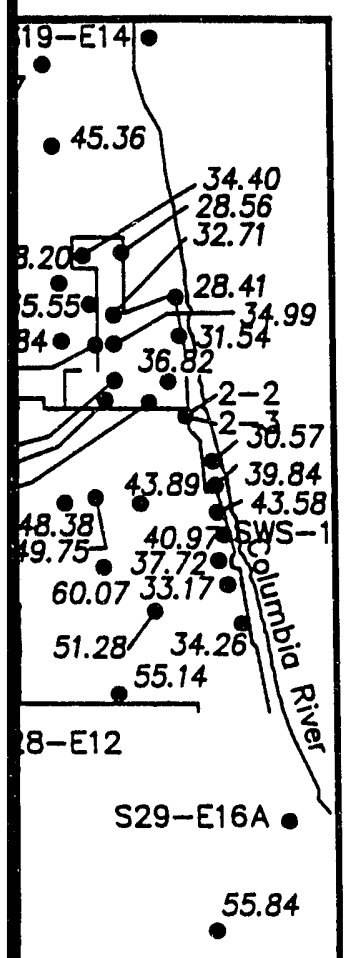

S31-E13

$\because S 32-E 13 A$

S32-E13B

$30.68 \bullet$

S34-E15

S36-E13A

47.63

.57 .24

53.19

$\bullet 50.30$

$\bullet 58.25$

52.53

연

91

GEOSCI \101692-F
300/1100 Area

Depth-to-Water Map June 1992

36.14 Depth to water in feet, as measured from well reference mark (generaily top of casing) to ground water surface

The 300 Area depth-to-water map has been prepared by the Geosciences Group, Environmental Division, of Westinghouse Hanford Company.

Note: To convert to metric, multiply elevation ( $\mathrm{ft}$ ) by 0.3048 to obtain elevation (m).

0 Mile

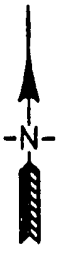




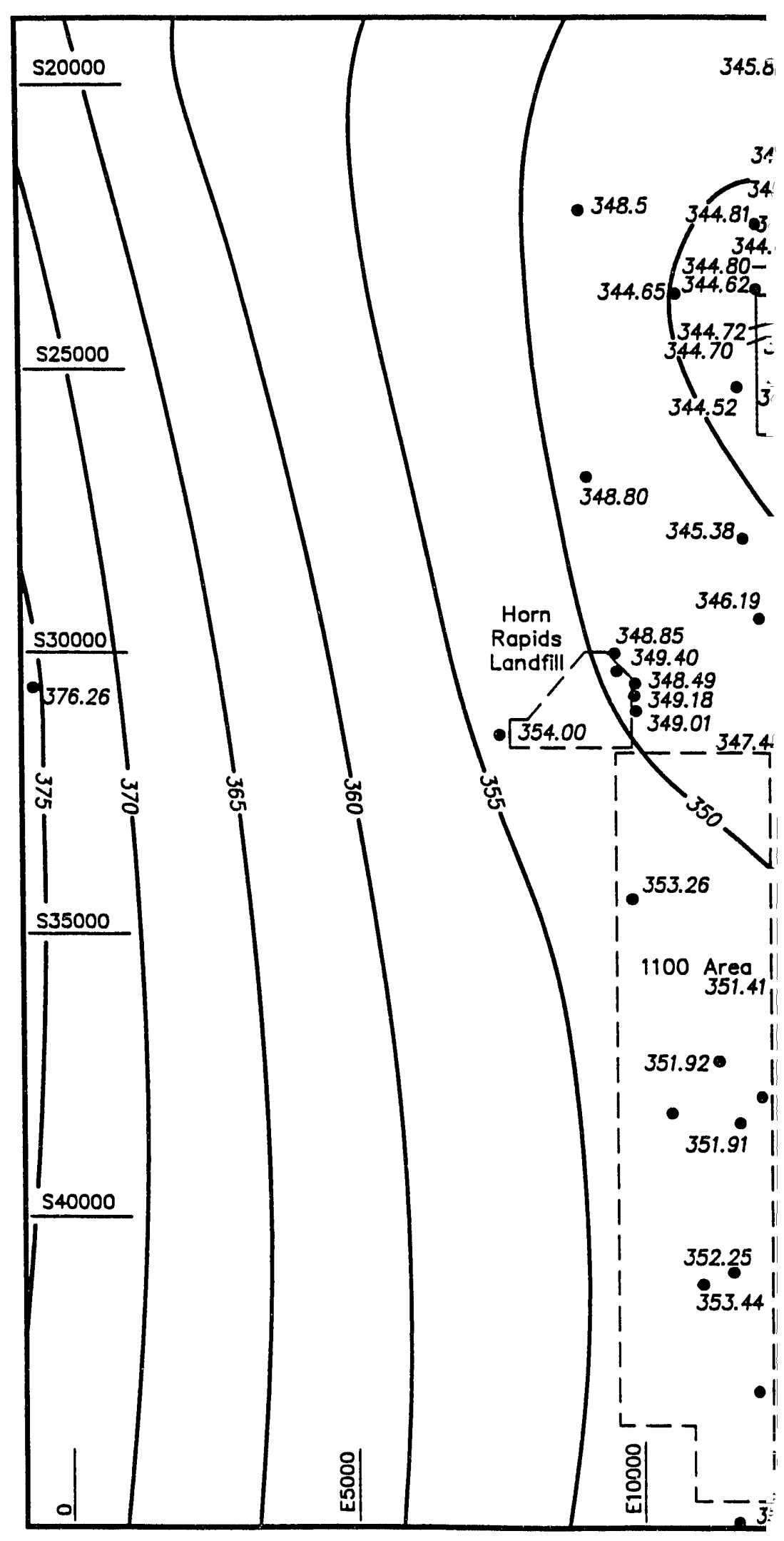




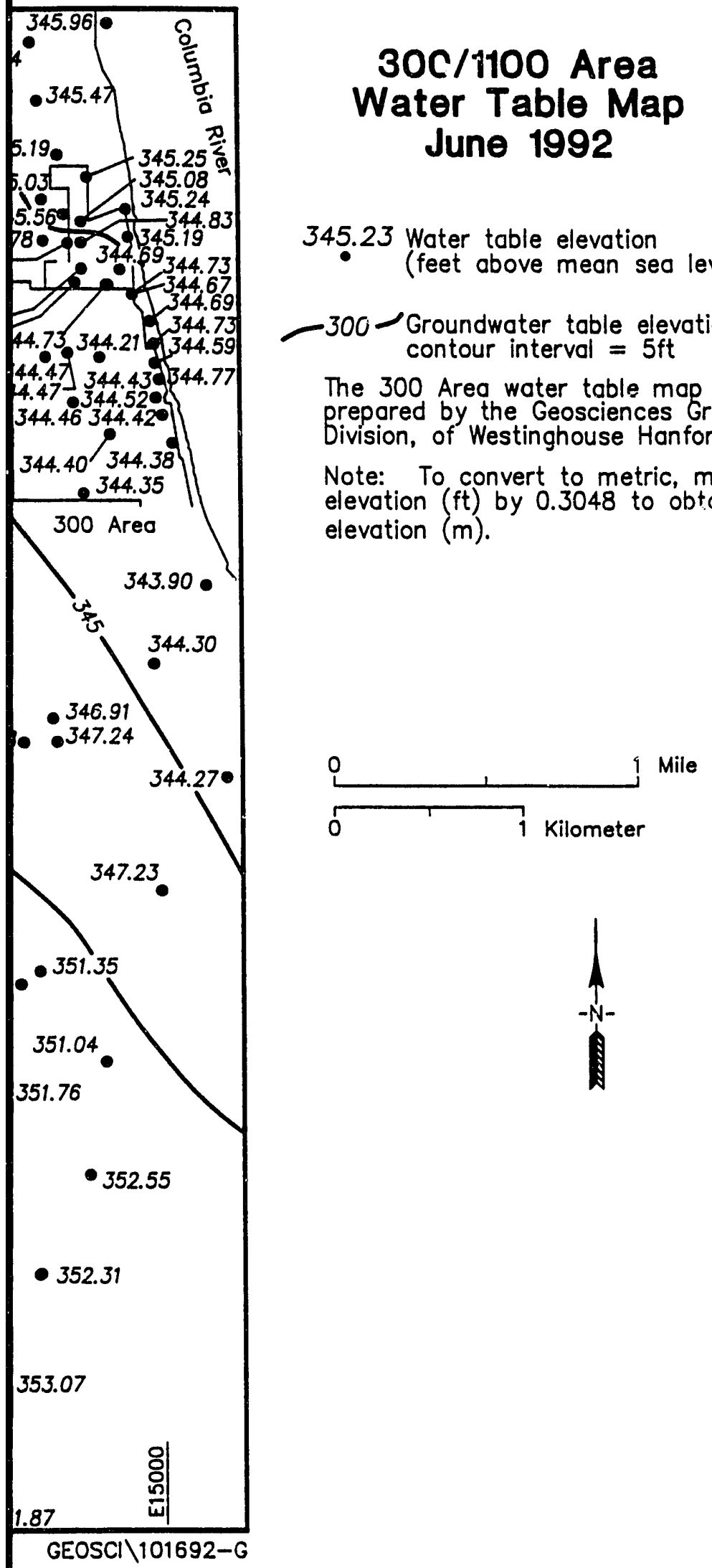

Figure 13 
WHC-EP-0394-5

APPENDIX A

WATER LEVEL MEASUREMENT DATA 
June 1992 Water Level Measurement Data (Sheet 1 of 21)

\begin{tabular}{|c|c|c|c|c|}
\hline \multirow{2}{*}{ Well } & \multirow{2}{*}{ Notes } & \multirow{2}{*}{$\begin{array}{l}\text { Depth to } \\
\text { Water, ft }\end{array}$} & \multicolumn{2}{|c|}{ Elevation, ft above msl } \\
\hline & & & Adjusted Casing & Water Level \\
\hline $\begin{array}{l}1-\mathrm{B} 2-12 \\
1-\mathrm{B} 2-13 \\
1-\mathrm{B} 3-1 \\
1-\mathrm{B} 3-46 \\
1-\mathrm{B} 3-47\end{array}$ & $\begin{array}{l}N \\
N \\
N \\
N\end{array}$ & $\begin{array}{l}41.97 \\
21.42 \\
45.16 \\
45.63 \\
42.59\end{array}$ & $\begin{array}{c}a \\
a \\
439.79 \\
a \\
a\end{array}$ & 394.63 \\
\hline $\begin{array}{l}1-B 4-1 \\
1-B 4-3 \\
1-B 4-4 \\
1-B 4-6 \\
1-84-8\end{array}$ & $\begin{array}{l}N \\
N\end{array}$ & $\begin{array}{l}63.26 \\
63.69 \\
75.32 \\
83.44 \\
75.10\end{array}$ & $\begin{array}{c}461.80 \\
461.75 \\
472.14 \\
\text { a } \\
\text { a }\end{array}$ & $\begin{array}{l}398.54 \\
398.06 \\
396.82\end{array}$ \\
\hline $\begin{array}{l}1-B 4-9 \\
1-B 5-1 \\
1-B 5-2 \\
1-B 8-6 \\
1-B 9-1\end{array}$ & $\begin{array}{l}N \\
N \\
N \\
Q\end{array}$ & $\begin{array}{l}72.93 \\
57.77 \\
60.58 \\
76.81 \\
97.87\end{array}$ & $\begin{array}{c}\text { a } \\
455.56 \\
a \\
a \\
478.87\end{array}$ & $\begin{array}{l}397.79 \\
381.00\end{array}$ \\
\hline $\begin{array}{l}1-B 9-2 \\
1-B 9-3 \\
1-D 2-5 \\
1-D 5-12 \\
1-D 5-13\end{array}$ & $\begin{array}{l}N \\
N\end{array}$ & $\begin{array}{l}98.87 \\
94.49 \\
74.54 \\
84.20 \\
86.15\end{array}$ & $\begin{array}{c}\text { a } \\
\text { a } \\
460.87 \\
469.67 \\
470.88\end{array}$ & $\begin{array}{l}386.33 \\
385.47 \\
384.73\end{array}$ \\
\hline $\begin{array}{l}1-D 5-16 \\
1-D 5-17 \\
1-D 5-18 \\
1-D 5-19 \\
1-D 5-20\end{array}$ & $\begin{array}{l}N \\
N \\
N \\
N \\
N\end{array}$ & $\begin{array}{l}88.19 \\
83.97 \\
81.49 \\
79.31 \\
82.57\end{array}$ & $\begin{array}{l}a \\
a \\
a \\
a \\
a\end{array}$ & \\
\hline $\begin{array}{l}1-08-3 \\
1-D 8-4 \\
1-D 8-5 \\
1-D 8-53 \\
1-D 8-54 A\end{array}$ & $\begin{array}{c}N-C \\
C \\
i \\
N\end{array}$ & $\begin{array}{l}65.10 \\
83.34 \\
67.69 \\
51.65 \\
58.37\end{array}$ & $\begin{array}{c}449.06 \\
468.13 \\
451.90 \\
\text { a } \\
\text { a }\end{array}$ & $\begin{array}{l}383.96 \\
384.79 \\
384.21\end{array}$ \\
\hline $\begin{array}{l}1-D 8-54 B \\
1-D 8-55 \\
1-D 8-6 \\
1-F 5-1 \\
1-F 5-3\end{array}$ & $\begin{array}{l}N \\
N \\
C \\
N\end{array}$ & $\begin{array}{l}57.67 \\
54.69 \\
91.07 \\
34.78 \\
37.99\end{array}$ & $\begin{array}{c}a \\
a \\
475.90 \\
406.56 \\
408.62\end{array}$ & $\begin{array}{l}384.83 \\
371.78 \\
370.63\end{array}$ \\
\hline
\end{tabular}


June 1992 Water Level Measurement Data (Sheet 2 of 21)

\begin{tabular}{|c|c|c|c|c|}
\hline \multirow{2}{*}{ Well } & \multirow{2}{*}{ Notes } & \multirow{2}{*}{$\begin{array}{l}\text { Depth to } \\
\text { Water, ft }\end{array}$} & \multicolumn{2}{|c|}{ Elevation, ft above ms 1} \\
\hline & & & Adjusted Casing & Water level \\
\hline $\begin{array}{l}1-F 7-1 \\
1-F 8-1 \\
1-H 3-1 \\
1-H 3-2 A \\
1-H 3-2 B\end{array}$ & $\begin{array}{l}N \\
N\end{array}$ & $\begin{array}{l}14.89 \\
34.77 \\
45.01 \\
41.62 \\
42.22\end{array}$ & $\begin{array}{l}389.74 \\
405.86 \\
421.48 \\
417.83 \\
418.42\end{array}$ & $\begin{array}{l}374.85 \\
371.09 \\
376.47 \\
376.21 \\
376.20\end{array}$ \\
\hline $\begin{array}{l}1-H 3-2 C \\
1-H 4-10 \\
1-H 4-11 \\
1-H 4-12 A \\
1-H 4-12 B\end{array}$ & $\begin{array}{l}N \\
N \\
N \\
N \\
N\end{array}$ & $\begin{array}{l}42.10 \\
29.68 \\
42.36 \\
38.84 \\
38.83\end{array}$ & $\begin{array}{l}418.22 \\
404.44 \\
416.84 \\
413.50 \\
413.52\end{array}$ & $\begin{array}{l}376.12 \\
374.76 \\
374.48 \\
374.66 \\
374.69\end{array}$ \\
\hline $\begin{array}{l}1-\mathrm{H} 4-12 \mathrm{C} \\
1-\mathrm{H} 4-13 \\
1-\mathrm{H} 4-14 \\
1-\mathrm{H} 4-15 \mathrm{~A} \\
1-\mathrm{H} 4-15 \mathrm{~B}\end{array}$ & $\begin{array}{l}N \\
N \\
N \\
N \\
N\end{array}$ & $\begin{array}{l}38.51 \\
43.76 \\
44.64 \\
32.42 \\
32.14\end{array}$ & $\begin{array}{l}413.52 \\
418.20 \\
420.59 \\
407.21 \\
406.92\end{array}$ & $\begin{array}{l}375.01 \\
374.44 \\
375.95 \\
374.79 \\
374.78\end{array}$ \\
\hline $\begin{array}{l}1-H 4-15 C Q \\
1-H 4-15 C R \\
1-H 4-15 C S \\
1-H 4-16 \\
1-H 4-17\end{array}$ & $\begin{array}{l}N \\
N \\
N \\
N\end{array}$ & $\begin{array}{l}15.46 \\
32.58 \\
32.40 \\
48.80 \\
44.24\end{array}$ & $\begin{array}{l}407.27 \\
407.37 \\
407.44 \\
424.23 \\
419.09\end{array}$ & $\begin{array}{l}391.81 \\
374.79 \\
375.04 \\
375.43 \\
374.85\end{array}$ \\
\hline $\begin{array}{l}1-H 4-18 \\
1-H 4-3 \\
1-H 4-4 \\
1-H 4-5 \\
1-H 4-6\end{array}$ & $\begin{array}{l}N \\
N \\
N \\
N\end{array}$ & $\begin{array}{l}46.78 \\
45.46 \\
39.12 \\
41.76 \\
43.38\end{array}$ & $\begin{array}{l}421.82 \\
420.29 \\
413.70 \\
416.21 \\
419.58\end{array}$ & $\begin{array}{l}375.04 \\
374.83 \\
374.58 \\
374.45 \\
376.20\end{array}$ \\
\hline $\begin{array}{l}1-H 4-7 \\
1-H 4-8 \\
1-H 4-9 \\
1-K-11 \\
1-K-19\end{array}$ & $\begin{array}{l}N \\
N \\
N\end{array}$ & $\begin{array}{l}44.98 \\
44.70 \\
43.22 \\
73.62 \\
33.00\end{array}$ & $\begin{array}{l}420.59 \\
420.00 \\
418.08 \\
467.66 \\
422.17\end{array}$ & $\begin{array}{l}375.61 \\
375.30 \\
374.86 \\
394.04 \\
389.17\end{array}$ \\
\hline $\begin{array}{l}1-K-20 \\
1-N-14 \\
1-N-16 \\
1-N-17 \\
1-N-18\end{array}$ & $\begin{array}{l}N \\
N \\
N\end{array}$ & $\begin{array}{l}33.78 \\
66.64 \\
68.72 \\
73.86 \\
71.30\end{array}$ & $\begin{array}{l}422.57 \\
453.15 \\
456.70 \\
461.20 \\
458.50\end{array}$ & $\begin{array}{l}388.79 \\
386.51 \\
387.98 \\
387.34 \\
387.20\end{array}$ \\
\hline
\end{tabular}


June 1992 Water Level Measurement Data (Sheet 3 of 21)

\begin{tabular}{|c|c|c|c|c|}
\hline \multirow{2}{*}{ Well } & \multirow{2}{*}{ Notes } & \multirow{2}{*}{$\begin{array}{l}\text { Depth to } \\
\text { Water, ft }\end{array}$} & \multicolumn{2}{|c|}{ Elevation, $\mathrm{ft}$ above msl } \\
\hline & & & Adjusted Casing & Water Level \\
\hline $\begin{array}{l}1-N-19 \\
1-N-2 \\
1-N-20 \\
1-N-21 \\
1-N-23\end{array}$ & $\begin{array}{l}N \\
N \\
N \\
N \\
N\end{array}$ & $\begin{array}{l}66.68 \\
72.11 \\
68.40 \\
69.57 \\
68.80\end{array}$ & $\begin{array}{l}453.90 \\
459.83 \\
455.90 \\
457.00 \\
456.30\end{array}$ & $\begin{array}{l}387.22 \\
387.72 \\
387.50 \\
387.43 \\
387.50\end{array}$ \\
\hline $\begin{array}{l}1-N-26 \\
1-N-27 \\
1-N-29 \\
1-N-3 \\
1-N-32\end{array}$ & $\begin{array}{l}N \\
N \\
N \\
N\end{array}$ & $\begin{array}{l}68.64 \\
60.90 \\
76.65 \\
71.55 \\
74.26\end{array}$ & $\begin{array}{l}455.80 \\
449.08 \\
465.25 \\
459.45 \\
462.08\end{array}$ & $\begin{array}{l}387.16 \\
388.18 \\
388.60 \\
387.90 \\
387.82\end{array}$ \\
\hline $\begin{array}{l}1-N-33 \\
1-N-34 \\
1-N-40 \\
1-N-41 \\
1-N-42\end{array}$ & $\begin{array}{l}N \\
N \\
N \\
N \\
N\end{array}$ & $\begin{array}{l}72.28 \\
71.66 \\
69.61 \\
71.08 \\
68.36\end{array}$ & $\begin{array}{l}459.87 \\
459.63 \\
456.35 \\
457.59 \\
455.14\end{array}$ & $\begin{array}{l}387.59 \\
387.97 \\
386.74 \\
386.51 \\
386.78\end{array}$ \\
\hline $\begin{array}{l}1-N-49 \\
1-N-50 \\
1-N-51 \\
1-N-52 \\
1-N-54\end{array}$ & $\begin{array}{l}N \\
N \\
N \\
N\end{array}$ & $\begin{array}{l}64.32 \\
77.08 \\
76.05 \\
74.75 \\
69.94\end{array}$ & $\begin{array}{l}450.72 \\
453.36 \\
462.18 \\
463.70 \\
457.51\end{array}$ & $\begin{array}{l}386.40 \\
376.28 \\
386.13 \\
388.95 \\
387.57\end{array}$ \\
\hline $\begin{array}{l}1-N-55 \\
1-N-56 \\
1-N-57 \\
1-N-59 \\
1-N-60\end{array}$ & $\begin{array}{l}N \\
N \\
N \\
N\end{array}$ & $\begin{array}{l}70.26 \\
70.85 \\
69.57 \\
70.55 \\
72.75\end{array}$ & $\begin{array}{l}457.85 \\
458.09 \\
457.76 \\
459.53 \\
461.94\end{array}$ & $\begin{array}{l}387.59 \\
387.24 \\
388.19 \\
388.98 \\
389.19\end{array}$ \\
\hline $\begin{array}{l}1-N-62 \\
1-N-63 \\
1-N-64 \\
1-N-65 \\
1-N-66\end{array}$ & $\begin{array}{l}N \\
N \\
N \\
N \\
N\end{array}$ & $\begin{array}{l}74.91 \\
78.20 \\
66.40 \\
68.57 \\
77.71\end{array}$ & $\begin{array}{l}463.59 \\
466.70 \\
454.63 \\
456.44 \\
465.25\end{array}$ & $\begin{array}{l}388.68 \\
388.50 \\
388.23 \\
387.87 \\
387.54\end{array}$ \\
\hline $\begin{array}{l}1-N-67 \\
1-N-69 \\
1-N-70 \\
1-N-71 \\
1-N-72\end{array}$ & $\begin{array}{l}N \\
N \\
N \\
C \\
N C\end{array}$ & $\begin{array}{l}71.27 \\
71.67 \\
67.28 \\
72.74 \\
68.45\end{array}$ & $\begin{array}{l}458.46 \\
458.84 \\
454.21 \\
462.50 \\
458.45\end{array}$ & $\begin{array}{l}387.19 \\
387.17 \\
386.93 \\
389.76 \\
390.00\end{array}$ \\
\hline
\end{tabular}


June 1992 Water Level Measurement Data (Sheet 4 of 21)

\begin{tabular}{|c|c|c|c|c|}
\hline \multirow{2}{*}{ Well } & \multirow{2}{*}{ Notes } & \multirow{2}{*}{$\begin{array}{l}\text { Depth to } \\
\text { Water, ft }\end{array}$} & \multicolumn{2}{|c|}{ Elevation, ft above msl } \\
\hline & & & Adjusted Casing & Water Level \\
\hline $\begin{array}{l}1-N-73 \\
1-N-74 \\
1-N-8 S \\
11-34-13 \\
11-41-13 C\end{array}$ & $\begin{array}{l}N C \\
C\end{array}$ & $\begin{array}{l}73.51 \\
67.53 \\
17.61 \\
42.91 \\
54.07\end{array}$ & $\begin{array}{l}462.74 \\
457.13 \\
404.57 \\
394.78 \\
405.98\end{array}$ & $\begin{array}{l}389.23 \\
389.60 \\
386.96 \\
351.87 \\
351.91\end{array}$ \\
\hline $\begin{array}{l}2-E 13-10 \\
2-E 13-12 \\
2-E 13-14 \\
2-E 16-1 \\
2-E 17-1\end{array}$ & $Q$ & $\begin{array}{l}335.72 \\
330.04 \\
343.05 \\
288.97 \\
316.88\end{array}$ & $\begin{array}{l}738.84 \\
731.34 \\
745.15 \\
696.44 \\
719.17\end{array}$ & $\begin{array}{l}403.12 \\
401.30 \\
402.10 \\
407.47 \\
402.29\end{array}$ \\
\hline $\begin{array}{l}2-E 17-10 \\
2-E 17-12 \\
2-E 17-13 \\
2-E 17-14 \\
2-E 17-15\end{array}$ & & $\begin{array}{l}312.16 \\
319.18 \\
316.81 \\
319.60 \\
320.08\end{array}$ & $\begin{array}{l}714.74 \\
721.70 \\
719.25 \\
722.18 \\
721.78\end{array}$ & $\begin{array}{l}402.58 \\
402.52 \\
402.44 \\
402.58 \\
401.70\end{array}$ \\
\hline $\begin{array}{l}2-E 17-16 \\
2-E 17-17 \\
2-E 17-18 \\
2-E 17-19 \\
2-E 17-20\end{array}$ & $N$ & $\begin{array}{l}318.26 \\
317.45 \\
318.19 \\
317.33 \\
316.68\end{array}$ & $\begin{array}{l}720.58 \\
719.92 \\
720.65 \\
719.33 \\
719.23\end{array}$ & $\begin{array}{l}402.32 \\
402.47 \\
402.46 \\
402.00 \\
402.55\end{array}$ \\
\hline $\begin{array}{l}2-E 17-5 \\
2-E 17-6 \\
2-E 17-8 \\
2-E 17-9 \\
2-E 18-1\end{array}$ & & $\begin{array}{l}316.61 \\
317.60 \\
316.29 \\
316.28 \\
317.53\end{array}$ & $\begin{array}{l}718.69 \\
720.10 \\
718.38 \\
717.64 \\
720.24\end{array}$ & $\begin{array}{l}402.08 \\
402.50 \\
402.09 \\
401.36 \\
402.71\end{array}$ \\
\hline $\begin{array}{l}2-E 18-3 \\
2-E 18-4 \\
2-E 23-1 \\
2-E 23-2 \\
2-E 24-13\end{array}$ & N & $\begin{array}{l}319.42 \\
318.81 \\
306.62 \\
317.92 \\
288.49\end{array}$ & $\begin{array}{l}722.04 \\
721.57 \\
709.65 \\
720.64 \\
691.13\end{array}$ & $\begin{array}{l}402.62 \\
402.76 \\
403.03 \\
402.72 \\
402.64\end{array}$ \\
\hline $\begin{array}{l}2-E 24-14 \\
2-E 24-16 \\
2-E 24-17 \\
2-E 24-18 \\
2-E 24-19\end{array}$ & & $\begin{array}{l}288.63 \\
315.61 \\
317.08 \\
316.80 \\
291.07\end{array}$ & $\begin{array}{l}691.31 \\
718.27 \\
718.69 \\
719.28 \\
693.65\end{array}$ & $\begin{array}{l}402.68 \\
402.66 \\
401.61 \\
402.48 \\
402.58\end{array}$ \\
\hline
\end{tabular}


June 1992 Water Leve1 Measurement Data (Sheet 5 of 21)

\begin{tabular}{|c|c|c|c|c|}
\hline \multirow{2}{*}{ Well } & \multirow{2}{*}{ Notes } & \multirow{2}{*}{$\begin{array}{l}\text { Depth to } \\
\text { Water, ft }\end{array}$} & \multicolumn{2}{|c|}{ Elevation, ft above msl } \\
\hline & & & Adjusted Casing & Water Level \\
\hline $\begin{array}{l}2-E 24-2 \\
2-E 24-20 \\
2-E 24-7 \\
2-E 24-8 \\
2-E 25-1\end{array}$ & $N$ & $\begin{array}{l}314.87 \\
286.38 \\
313.26 \\
285.83 \\
287.83\end{array}$ & $\begin{array}{l}717.47 \\
689.02 \\
716.01 \\
688.81 \\
690.57\end{array}$ & $\begin{array}{l}402.60 \\
402.64 \\
402.75 \\
402.98 \\
402.74\end{array}$ \\
\hline $\begin{array}{l}2-E 25-10 \\
2-E 25-11 \\
2-E 25-13 \\
2-E 25-15 \\
2-E 25-18\end{array}$ & N & $\begin{array}{l}253.03 \\
278.56 \\
279.70 \\
286.99 \\
276.24\end{array}$ & $\begin{array}{l}655.84 \\
681.28 \\
682.43 \\
689.73 \\
679.05\end{array}$ & $\begin{array}{l}402.81 \\
402.72 \\
402.73 \\
402.74 \\
402.81\end{array}$ \\
\hline $\begin{array}{l}2-E 25-19 \\
2-E 25-2 \\
2-E 25-20 \\
2-E 25-24 \\
2-E 25-25\end{array}$ & & $\begin{array}{l}274.57 \\
272.63 \\
273.65 \\
276.53 \\
266.07\end{array}$ & $\begin{array}{l}677.20 \\
675.45 \\
676.30 \\
679.55 \\
669.42\end{array}$ & $\begin{array}{l}402.63 \\
402.82 \\
402.65 \\
403.02 \\
403.35\end{array}$ \\
\hline $\begin{array}{l}2-E 25-26 \\
2-E 25-27 \\
2-E 25-28 \\
2-E 25-29 Q \\
2-E 25-30 Q\end{array}$ & & $\begin{array}{l}265.60 \\
272.95 \\
259.35 \\
269.65 \\
275.30\end{array}$ & $\begin{array}{l}668.52 \\
676.08 \\
662.44 \\
672.62 \\
677.91\end{array}$ & $\begin{array}{l}402.92 \\
403.13 \\
403.09 \\
402.97 \\
402.61\end{array}$ \\
\hline $\begin{array}{l}2-E 25-31 \\
2-E 25-32 P \\
2-E 25-32 Q \\
2-E 25-34 \\
2-E 25-35\end{array}$ & & $\begin{array}{l}269.63 \\
267.51 \\
267.31 \\
259.88 \\
271.65\end{array}$ & $\begin{array}{l}672.53 \\
670.04 \\
670.04 \\
662.87 \\
674.39\end{array}$ & $\begin{array}{l}402.90 \\
402.53 \\
402.73 \\
402.99 \\
402.74\end{array}$ \\
\hline $\begin{array}{l}2-E 25-36 \\
2-E 25-37 \\
2-E 25-38 \\
2-E 25-39 \\
2-E 25-4\end{array}$ & N & $\begin{array}{l}304.90 \\
270.46 \\
270.53 \\
267.73 \\
258.04\end{array}$ & $\begin{array}{l}707.39 \\
673.29 \\
673.52 \\
671.23 \\
659.39\end{array}$ & $\begin{array}{l}402.49 \\
402.83 \\
402.99 \\
403.50 \\
401.35\end{array}$ \\
\hline $\begin{array}{l}2-E 25-40 \\
2-E 25-41 \\
2-E 25-42 \\
2-E 25-43 \\
2-E 25-6\end{array}$ & $\begin{array}{l}N \\
N\end{array}$ & $\begin{array}{l}262.96 \\
268.45 \\
280.46 \\
246.65 \\
258.10\end{array}$ & $\begin{array}{l}665.71 \\
671.26 \\
683.06 \\
649.66 \\
658.31\end{array}$ & $\begin{array}{l}402.75 \\
402.81 \\
402.60 \\
403.01 \\
400.21\end{array}$ \\
\hline
\end{tabular}


WHC-EP-0394-5

June 1992 Water Level Measurement Data

(Sheet 6 of 21)

\begin{tabular}{|c|c|c|c|c|}
\hline \multirow{2}{*}{ Well } & \multirow{2}{*}{ Notes } & \multirow{2}{*}{$\begin{array}{l}\text { Depth to } \\
\text { Water, ft }\end{array}$} & \multicolumn{2}{|c|}{ Elevation, ft above insl } \\
\hline & & & Adjusted Casing & Water Level \\
\hline $\begin{array}{l}2-E 25-9 \\
2-E 26-1 \\
2-E 26-10 \\
2-E 26-11 \\
2-E 26-12\end{array}$ & $\begin{array}{l}N \\
N \\
N\end{array}$ & $\begin{array}{l}254.15 \\
213.88 \\
198.24 \\
194.08 \\
227.33\end{array}$ & $\begin{array}{l}654.86 \\
617.25 \\
601.47 \\
599.68 \\
630.51\end{array}$ & $\begin{array}{l}400.71 \\
403.37 \\
403.23 \\
405.60 \\
403.18\end{array}$ \\
\hline $\begin{array}{l}2-E 26-13 \\
2-E 26-2 \\
2-E 26-4 \\
2-E 26-9 \\
2-E 27-10\end{array}$ & $N$ & $\begin{array}{l}201.68 \\
232.32 \\
244.84 \\
199.52 \\
221.54\end{array}$ & $\begin{array}{l}604.79 \\
635.30 \\
647.76 \\
602.89 \\
624.47\end{array}$ & $\begin{array}{l}403.11 \\
402.98 \\
402.92 \\
403.37 \\
402.93\end{array}$ \\
\hline $\begin{array}{l}2-E 27-11 \\
2-E 27-12 \\
2-E 27-13 \\
2-E 27-14 \\
2-E 27-15\end{array}$ & & $\begin{array}{l}240.87 \\
258.32 \\
266.20 \\
255.71 \\
250.39\end{array}$ & $\begin{array}{l}643.29 \\
660.95 \\
668.99 \\
658.34 \\
652.67\end{array}$ & $\begin{array}{l}402.42 \\
402.63 \\
402.79 \\
402.63 \\
402.28\end{array}$ \\
\hline $\begin{array}{l}2-E 27-16 \\
2-E 27-17 \\
2-E 27-7 \\
2-E 27-8 \\
2-E 27-9\end{array}$ & N & $\begin{array}{l}249.75 \\
231.94 \\
231.84 \\
235.26 \\
226.56\end{array}$ & $\begin{array}{l}652.13 \\
634.50 \\
634.67 \\
637.83 \\
629.21\end{array}$ & $\begin{array}{l}402.38 \\
402.56 \\
402.83 \\
402.57 \\
402.65\end{array}$ \\
\hline $\begin{array}{l}2-E 28-12 \\
2-E 28-17 \\
2-E 28-18 \\
2-E 28-26 \\
2-E 28-27\end{array}$ & & $\begin{array}{l}305.34 \\
304.79 \\
289.72 \\
284.82 \\
277.97\end{array}$ & $\begin{array}{l}708.60 \\
708.56 \\
692.58 \\
687.26 \\
680.37\end{array}$ & $\begin{array}{l}403.26 \\
403.77 \\
402.86 \\
402.44 \\
402.40\end{array}$ \\
\hline $\begin{array}{l}2-E 28-28 \\
2-E 28-4 \\
2-E 28-6 \\
2-E 28-7 \\
2-E 28-9\end{array}$ & & $\begin{array}{l}284.22 \\
288.50 \\
297.10 \\
283.72 \\
297.89\end{array}$ & $\begin{array}{l}686.55 \\
691.55 \\
700.11 \\
685.91 \\
700.77\end{array}$ & $\begin{array}{l}402.33 \\
403.05 \\
403.01 \\
402.19 \\
402.88\end{array}$ \\
\hline $\begin{array}{l}2-E 32-1 \\
2-E 32-2 \\
2-E 32-3 \\
2-E 32-4 \\
2-E 32-5\end{array}$ & & $\begin{array}{l}253.43 \\
267.79 \\
274.14 \\
283.64 \\
279.79\end{array}$ & $\begin{array}{l}656.17 \\
670.06 \\
676.51 \\
685.88 \\
682.14\end{array}$ & $\begin{array}{l}402.74 \\
402.27 \\
402.37 \\
402.24 \\
402.35\end{array}$ \\
\hline
\end{tabular}


June 1992 Water Level Measurement Data

(Sheet 7 of 21)

\begin{tabular}{|c|c|c|c|c|}
\hline \multirow{2}{*}{ Well } & \multirow{2}{*}{ Notes } & \multirow{2}{*}{$\begin{array}{l}\text { Depth to } \\
\text { Water, ft }\end{array}$} & \multicolumn{2}{|c|}{ Elevation, ft above msl } \\
\hline & & & Adjusted Casing & Water Level \\
\hline $\begin{array}{l}2-E 32-6 \\
2-E 32-7 \\
2-E 32-8 \\
2-E 32-9 \\
2-E 33-1\end{array}$ & $\begin{array}{l}N \\
N \\
N \\
N \\
Q\end{array}$ & $\begin{array}{l}264.90 \\
255.84 \\
243.10 \\
240.76 \\
229.39\end{array}$ & $\begin{array}{l}667.22 \\
658.42 \\
643.33 \\
643.07 \\
629.24\end{array}$ & $\begin{array}{l}402.32 \\
402.58 \\
400.23 \\
402.31 \\
399.35\end{array}$ \\
\hline $\begin{array}{l}2-E 33-12 \\
2-E 33-13 \\
2-E 33-14 \\
2-E 33-15 \\
2-E 33-2\end{array}$ & $\begin{array}{l}P \\
N\end{array}$ & $\begin{array}{l}219.48 \\
224.10 \\
219.23 \\
223.55 \\
229.01\end{array}$ & $\begin{array}{l}623.00 \\
625.53 \\
622.12 \\
627.40 \\
629.72\end{array}$ & $\begin{array}{l}403.52 \\
401.43 \\
402.89 \\
403.85 \\
400.71\end{array}$ \\
\hline $\begin{array}{l}2-E 33-21 \\
2-E 33-24 \\
2-E 33-26 \\
2-E 33-28 \\
2-E 33-29\end{array}$ & & $\begin{array}{l}265.59 \\
235.18 \\
231.20 \\
261.77 \\
271.36\end{array}$ & $\begin{array}{l}668.13 \\
637.70 \\
632.51 \\
664.23 \\
673.77\end{array}$ & $\begin{array}{l}402.54 \\
402.52 \\
401.31 \\
402.46 \\
402.41\end{array}$ \\
\hline $\begin{array}{l}2-E 33-3 \\
2-E 33-30 \\
2-E 33-31 \\
2-E 33-32 \\
2-E 33-33\end{array}$ & $Q$ & $\begin{array}{l}227.83 \\
261.24 \\
244.76 \\
257.42 \\
237.60\end{array}$ & $\begin{array}{l}625.73 \\
663.70 \\
647.28 \\
659.83 \\
640.17\end{array}$ & $\begin{array}{l}397.90 \\
402.46 \\
402.52 \\
402.41 \\
402.57\end{array}$ \\
\hline $\begin{array}{l}2-E 33-34 \\
?-E 33-35 \\
2-E 33-36 \\
2-E 33-37 \\
2-E 33-38\end{array}$ & N & $\begin{array}{l}230.98 \\
240.67 \\
245.54 \\
250.50 \\
229.52\end{array}$ & $\begin{array}{l}633.33 \\
643.01 \\
646.67 \\
653.01 \\
631.9\end{array}$ & $\begin{array}{l}402.35 \\
402.34 \\
401.13 \\
402.51 \\
402.43\end{array}$ \\
\hline $\begin{array}{l}2-E 33-39 \\
2-E 33-4 \\
2-E 33-40 \\
2-E 33-41 \\
2-E 33-42\end{array}$ & $\begin{array}{l}N \\
Q \\
N \\
N \\
N\end{array}$ & $\begin{array}{l}220.91 \\
227.12 \\
221.76 \\
252.23 \\
251.56\end{array}$ & $\begin{array}{l}623.32 \\
624.92 \\
624.32 \\
654.95 \\
654.08\end{array}$ & $\begin{array}{l}402.41 \\
397.80 \\
402.56 \\
402.72 \\
402.52\end{array}$ \\
\hline $\begin{array}{l}2-E 33-43 \\
2-F .33-5 \\
2-E 33-7 \\
2-E 33-8 \\
2-E 34-1\end{array}$ & $\begin{array}{l}N \\
N \\
N\end{array}$ & $\begin{array}{l}260.04 \\
231.89 \\
225.10 \\
248.18 \\
226.69\end{array}$ & $\begin{array}{l}662.46 \\
632.07 \\
626.58 \\
650.73 \\
629.42\end{array}$ & $\begin{array}{l}402.42 \\
400.18 \\
401.48 \\
402.55 \\
402.73\end{array}$ \\
\hline
\end{tabular}


June 1992 Water Level Measurement Data

(Sheet 8 of 21)

\begin{tabular}{|c|c|c|c|c|}
\hline \multirow{2}{*}{ Well } & \multirow{2}{*}{ Notes } & \multirow{2}{*}{$\begin{array}{l}\text { Depth to } \\
\text { Water, ft }\end{array}$} & \multicolumn{2}{|c|}{ Elevation, $\mathrm{ft}$ above msl } \\
\hline & & & Adjusted Casing & Water Level \\
\hline $\begin{array}{l}2-E 34-10 \\
2-E 34-11 \\
2-E 34-2 \\
2-E 34-3 \\
2-E 34-5\end{array}$ & $\begin{array}{l}N \\
N\end{array}$ & $\begin{array}{l}237.02 \\
215.18 \\
228.28 \\
208.54 \\
186.96\end{array}$ & $\begin{array}{l}639.55 \\
630.80 \\
611.52 \\
590.79\end{array}$ & $\begin{array}{l}402.53 \\
402.52 \\
402.98 \\
403.83\end{array}$ \\
\hline $\begin{array}{l}2-E 34-6 \\
2-E 34-7 \\
2-E 34-8 \\
2-E 34-9 \\
2-E 35-1\end{array}$ & $N$ & $\begin{array}{l}195.26 \\
201.58 \\
238.33 \\
225.98 \\
194.99\end{array}$ & $\begin{array}{l}597.83 \\
604.27 \\
640.52 \\
628.47 \\
598.31\end{array}$ & $\begin{array}{l}402.57 \\
402.69 \\
402.19 \\
402.49 \\
403.32\end{array}$ \\
\hline $\begin{array}{l}2-E 35-2 \\
2-W 10-10 \\
2-W 10-11 \\
2-W 10-12 \\
2-W 10-13\end{array}$ & & $\begin{array}{l}198.68 \\
212.97 \\
212.85 \\
212.56 \\
235.71\end{array}$ & $\begin{array}{l}602.10 \\
675.09 \\
675.26 \\
674.75 \\
699.04\end{array}$ & $\begin{array}{l}403.42 \\
462.12 \\
462.41 \\
46 . .19 \\
463.33\end{array}$ \\
\hline $\begin{array}{l}2-W 10-14 \\
2-W 10-15 \\
2-W 10-16 \\
2-W 10-17 \\
2-W 10-18\end{array}$ & $\begin{array}{l}N \\
N\end{array}$ & $\begin{array}{l}236.52 \\
213.54 \\
210.12 \\
207.89 \\
? 07.17\end{array}$ & $\begin{array}{l}699.43 \\
675.64 \\
672.76 \\
670.59 \\
670.68\end{array}$ & $\begin{array}{l}462.91 \\
462.10 \\
462.64 \\
462.70 \\
463.51\end{array}$ \\
\hline $\begin{array}{l}2-W 10-2 \\
2-W 10-3 \\
2-W 10-5 \\
2-W 10-8 \\
2-W 11-10\end{array}$ & & $\begin{array}{l}211.36 \\
208.77 \\
208.58 \\
218.21 \\
274.00\end{array}$ & $\begin{array}{l}673.99 \\
671.46 \\
672.31 \\
680.55 \\
728.89\end{array}$ & $\begin{array}{l}462.67 \\
462.69 \\
463.73 \\
461.84 \\
454.89\end{array}$ \\
\hline $\begin{array}{l}2-W 11-12 \\
2-W 11-14 \\
2-W 11-19 \\
2-W 11-23 \\
2-W 11-24\end{array}$ & $N$ & $\begin{array}{l}216.73 \\
256.29 \\
248.91 \\
223.92 \\
225.16\end{array}$ & $\begin{array}{l}679.26 \\
715.16 \\
707.00 \\
685.42 \\
687.17\end{array}$ & $\begin{array}{l}462.53 \\
458.87 \\
458.09 \\
461.50 \\
462.01\end{array}$ \\
\hline $\begin{array}{l}2-W 11-27 \\
2-W 11-28 \\
2-W 11-6 \\
2-W 11-7 \\
2-W 11-9\end{array}$ & $\begin{array}{l}N \\
N\end{array}$ & $\begin{array}{l}223.24 \\
231.44 \\
256.98 \\
248.04 \\
265.72\end{array}$ & $\begin{array}{l}685.01 \\
693.22 \\
716.23 \\
709.11 \\
722.94\end{array}$ & $\begin{array}{l}461.77 \\
461.78 \\
459.25 \\
461.07 \\
457.22\end{array}$ \\
\hline
\end{tabular}


WHC-EP-0394-5

June 1992 Water Level Measurement Data

(Sheet 9 of 21)

\begin{tabular}{|c|c|c|c|c|}
\hline \multirow{2}{*}{ Well } & \multirow{2}{*}{ Notes } & \multirow{2}{*}{$\begin{array}{l}\text { Depth to } \\
\text { Water, ft }\end{array}$} & \multicolumn{2}{|c|}{ Elevation, ft above msl } \\
\hline & & & Adjusted Casing & Water Level \\
\hline $\begin{array}{l}2-W 12-1 \\
2-W 14-1 \\
2-W 14-12 \\
2-W 14-9 \\
2-W 15-10\end{array}$ & $\begin{array}{l}N \\
N\end{array}$ & $\begin{array}{l}276.12 \\
204.57 \\
206.02 \\
223.13 \\
211.66\end{array}$ & $\begin{array}{c}726.46 \\
665.83 \\
670.25 \\
a \\
676.00\end{array}$ & $\begin{array}{l}450.34 \\
461.26 \\
464.23 \\
464.34\end{array}$ \\
\hline $\begin{array}{l}2-W 15-12 \\
2-W 15-13 \\
2-W 15-15 \\
2-W 15-16 \\
2-W 15-17\end{array}$ & $\begin{array}{l}N \\
N \\
N \\
N\end{array}$ & $\begin{array}{l}205.99 \\
206.19 \\
232.64 \\
218.96 \\
219.12\end{array}$ & $\begin{array}{l}670.07 \\
670.12 \\
697.96 \\
684.89 \\
684.64\end{array}$ & $\begin{array}{l}464.08 \\
463.93 \\
465.32 \\
465.93 \\
465.52\end{array}$ \\
\hline $\begin{array}{l}2-W 15-18 \\
2-W 15-19 \\
2-W 15-20 \\
2-W 15-22 \\
2-W 15-23\end{array}$ & $N$ & $\begin{array}{l}219.54 \\
226.77 \\
233.18 \\
204.70 \\
234.38\end{array}$ & $\begin{array}{l}685.71 \\
691.60 \\
698.36 \\
670.52 \\
699.49\end{array}$ & $\begin{array}{l}466.17 \\
464.83 \\
464.88 \\
465.82 \\
465.11\end{array}$ \\
\hline $\begin{array}{l}2-W 15-24 \\
2-W 15-3 \\
2-W 15-4 \\
2-W 15-5 \\
2-W 18-21\end{array}$ & $\begin{array}{l}N \\
N\end{array}$ & $\begin{array}{l}233.87 \\
206.39 \\
196.36 \\
203.18 \\
202.06\end{array}$ & $\begin{array}{l}699.37 \\
670.50 \\
662.00 \\
670.68 \\
668.62\end{array}$ & $\begin{array}{l}465.50 \\
464.11 \\
465.64 \\
467.50 \\
466.56\end{array}$ \\
\hline $\begin{array}{l}2-W 18-22 \\
2-W 18-23 \\
2-W 18-24 \\
2-W 18-25 \\
2-W 18-26\end{array}$ & $\begin{array}{l}N \\
N\end{array}$ & $\begin{array}{l}203.62 \\
231.26 \\
217.96 \\
198.32 \\
233.51\end{array}$ & $\begin{array}{l}668.49 \\
696.81 \\
684.35 \\
665.80 \\
699.05\end{array}$ & $\begin{array}{l}464.87 \\
465.55 \\
466.39 \\
467.48 \\
465.54\end{array}$ \\
\hline $\begin{array}{l}2-W 18-27 \\
2-W 18-28 \\
2-W 18-29 \\
2-W 18-30 \\
2-W 18-31\end{array}$ & $\begin{array}{l}N \\
N \\
Q \\
N \\
N\end{array}$ & $\begin{array}{l}224.64 \\
214.15 \\
127.91 \\
205.93 \\
196.74\end{array}$ & $\begin{array}{l}689.99 \\
679.73 \\
673.88 \\
672.57 \\
663.90\end{array}$ & $\begin{array}{l}465.35 \\
465.58 \\
545.97 \\
466.64 \\
467.16\end{array}$ \\
\hline $\begin{array}{l}2-W 19-1 \\
2-W 19-12 \\
2-W 19-14 \\
2-W 19-15 \\
2-W 19-2\end{array}$ & $\begin{array}{l}N \\
N\end{array}$ & $\begin{array}{l}206.23 \\
206.32 \\
231.18 \\
230.62 \\
235.78\end{array}$ & $\begin{array}{l}673.77 \\
673.25 \\
693.21 \\
693.28 \\
694.04\end{array}$ & $\begin{array}{l}467.54 \\
466.93 \\
462.03 \\
462.66 \\
458.26\end{array}$ \\
\hline
\end{tabular}


June 1992 Water Level Measurement Data

(Sheet 10 of 21)

\begin{tabular}{|c|c|c|c|c|}
\hline \multirow{2}{*}{ Well } & \multirow{2}{*}{ Notes } & \multirow{2}{*}{$\begin{array}{l}\text { Depth to } \\
\text { Water, ft }\end{array}$} & \multicolumn{2}{|c|}{ Elevation, ft above ms 1} \\
\hline & & & Adjusted Casing & Water Level \\
\hline $\begin{array}{l}2-W 19-20 \\
2-W 19-21 \\
2-W 19-24 \\
2-W 19-27 \\
2-W 19-28\end{array}$ & & $\begin{array}{l}234.00 \\
211.16 \\
239.10 \\
217.03 \\
243.46\end{array}$ & $\begin{array}{l}691.04 \\
678.53 \\
696.95 \\
683.65 \\
703.09\end{array}$ & $\begin{array}{l}457.04 \\
467.37 \\
457.85 \\
466.62 \\
459.63\end{array}$ \\
\hline $\begin{array}{l}2-W 19-29 \\
2-W 19-31 \\
2-W 19-32 \\
2-W 19-4 \\
2-W 19-91\end{array}$ & $\begin{array}{l}N \\
N \\
N\end{array}$ & $\begin{array}{l}242.86 \\
207.34 \\
207.73 \\
257.24 \\
116.71\end{array}$ & $\begin{array}{l}701.87 \\
673.95 \\
674.65 \\
715.26 \\
677.90\end{array}$ & $\begin{array}{l}459.01 \\
466.61 \\
466.92 \\
458.02 \\
561.19\end{array}$ \\
\hline $\begin{array}{l}2-W 19-92 \\
2-W 19-93 \\
2-W 21-1 \\
2-W 22-17 \\
2-W 22-19\end{array}$ & $\begin{array}{l}N \\
N\end{array}$ & $\begin{array}{r}112.94 \\
94.33 \\
245.41 \\
210.35 \\
225.18\end{array}$ & $\begin{array}{l}677.90 \\
677.43 \\
699.26 \\
671.62 \\
681.26\end{array}$ & $\begin{array}{l}564.96 \\
583.10 \\
453.85 \\
461.27 \\
456.08\end{array}$ \\
\hline $\begin{array}{l}2-W 22-21 \\
2-W 22-22 \\
2-W 22-26 \\
2-W 22-28 \\
2-W 22-39\end{array}$ & $\begin{array}{l}N \\
N\end{array}$ & $\begin{array}{l}211.86 \\
232.46 \\
219.57 \\
230.94 \\
207.58\end{array}$ & $\begin{array}{l}670.00 \\
690.05 \\
680.30 \\
689.00 \\
668.00\end{array}$ & $\begin{array}{l}458.14 \\
457.59 \\
460.73 \\
458.06 \\
460.42\end{array}$ \\
\hline $\begin{array}{l}2-W 22-40 \\
2-W 22-41 \\
2-W 22-42 \\
2-W 22-43 \\
2-W 22-44\end{array}$ & N & $\begin{array}{l}235.11 \\
234.67 \\
234.27 \\
233.29 \\
215.03\end{array}$ & $\begin{array}{l}692.23 \\
691.74 \\
691.16 \\
691.35 \\
677.87\end{array}$ & $\begin{array}{l}457.12 \\
457.07 \\
456.89 \\
458.06 \\
462.84\end{array}$ \\
\hline $\begin{array}{l}2-W 22-46 \\
2-W 22-7 \\
2-W 23-1 \\
2-W 23-11 \\
2-W 23-12\end{array}$ & $\begin{array}{l}N \\
N \\
N\end{array}$ & $\begin{array}{l}210.84 \\
229.38 \\
202.46 \\
200.68 \\
202.74\end{array}$ & $\begin{array}{l}670.92 \\
687.41 \\
666.14 \\
664.14 \\
666.15\end{array}$ & $\begin{array}{l}460.08 \\
458.03 \\
463.68 \\
463.46 \\
463.41\end{array}$ \\
\hline $\begin{array}{l}2-W 23-13 \\
2-W 23-14 \\
2-W 23-15 \\
2-W 23-2 \\
2-W 23-3\end{array}$ & $\begin{array}{l}N \\
N \\
N \\
N \\
N\end{array}$ & $\begin{array}{l}202.18 \\
200.90 \\
193.92 \\
201.42 \\
202.55\end{array}$ & $\begin{array}{l}666.09 \\
663.74 \\
655.18 \\
663.48 \\
663.56\end{array}$ & $\begin{array}{l}463.91 \\
462.84 \\
461.26 \\
462.06 \\
461.01\end{array}$ \\
\hline
\end{tabular}


June 1992 Water Level Measurement Data (Sheet 11 of 21)

\begin{tabular}{|c|c|c|c|c|}
\hline \multirow{2}{*}{ Well } & \multirow{2}{*}{ Notes } & \multirow{2}{*}{$\begin{array}{l}\text { Depth to } \\
\text { Water, ft }\end{array}$} & \multicolumn{2}{|c|}{ Elevation, $\mathrm{ft}$ above msl } \\
\hline & & & Adjusted Casing & Water Level \\
\hline $\begin{array}{l}2-W 23-4 \\
2-W 23-6 \\
2-W 23-7 \\
2-W 23-8 \\
2-W 26-8\end{array}$ & $\begin{array}{l}N \\
N \\
N\end{array}$ & $\begin{array}{l}198.05 \\
206.36 \\
201.04 \\
200.84 \\
206.87\end{array}$ & $\begin{array}{l}662.82 \\
666.76 \\
663.67 \\
663.95 \\
666.31\end{array}$ & $\begin{array}{l}464.77 \\
460.40 \\
462.63 \\
463.11 \\
459.44\end{array}$ \\
\hline $\begin{array}{l}2-W 26-9 \\
2-W 6-1 \\
2-W 6-2 \\
2-W 6-3 \\
2-W 6-4\end{array}$ & $\begin{array}{l}N \\
N \\
N\end{array}$ & $\begin{array}{l}195.40 \\
245.85 \\
233.95 \\
241.94 \\
242.83\end{array}$ & $\begin{array}{l}654.16 \\
702.53 \\
692.45 \\
699.57 \\
700.99\end{array}$ & $\begin{array}{l}458.76 \\
456.68 \\
458.50 \\
457.63 \\
458.16\end{array}$ \\
\hline $\begin{array}{l}2-W 6-6 \\
2-W 6-7 \\
2-W 6-8 \\
2-W 7-1 \\
2-W 7-10\end{array}$ & $\begin{array}{l}N \\
N \\
N \\
N\end{array}$ & $\begin{array}{l}255.06 \\
255.39 \\
238.45 \\
231.45 \\
231.80\end{array}$ & $\begin{array}{l}694.21 \\
694.47 \\
710.02 \\
690.71 \\
689.66\end{array}$ & $\begin{array}{l}439.15 \\
439.08 \\
471.57 \\
459.26 \\
457.86\end{array}$ \\
\hline $\begin{array}{l}2-W 7-11 \\
2-W 7-12 \\
2-W 7-2 \\
2-W 7-3 \\
2-W 7-4\end{array}$ & $\begin{array}{l}N \\
N \\
N\end{array}$ & $\begin{array}{l}222.49 \\
228.67 \\
217.44 \\
219.11 \\
211.66\end{array}$ & $\begin{array}{l}681.19 \\
687.67 \\
675.59 \\
676.14 \\
671.69\end{array}$ & $\begin{array}{l}458.70 \\
459.00 \\
458.15 \\
457.03 \\
460.03\end{array}$ \\
\hline $\begin{array}{l}2-W 7-5 \\
2-W 7-6 \\
2-W 7-7 \\
2-W 7-8 \\
2-W 7-9\end{array}$ & & $\begin{array}{l}215.37 \\
221.13 \\
217.22 \\
231.03 \\
232.41\end{array}$ & $\begin{array}{l}673.05 \\
678.64 \\
674.94 \\
687.35 \\
692.09\end{array}$ & $\begin{array}{l}457.68 \\
457.51 \\
457.72 \\
456.32 \\
459.68\end{array}$ \\
\hline $\begin{array}{l}2-W 8-1 \\
2-W 9-1 \\
3-1-1 \\
3-1-10 \\
3-1-11\end{array}$ & & $\begin{array}{r}241.64 \\
275.43 \\
31.54 \\
28.41 \\
32.71\end{array}$ & $\begin{array}{l}701.33 \\
737.73 \\
376.73 \\
373.65 \\
377.79\end{array}$ & $\begin{array}{l}459.69 \\
462.30 \\
345.19 \\
345.24 \\
345.08\end{array}$ \\
\hline $\begin{array}{l}3-1-12 \\
3-1-13 \\
3-1-14 \\
3-1-15 \\
3-1-16 A\end{array}$ & & $\begin{array}{l}39.65 \\
43.84 \\
38.20 \\
34.40 \\
36.82\end{array}$ & $\begin{array}{l}384.45 \\
388.62 \\
383.23 \\
379.59 \\
381.51\end{array}$ & $\begin{array}{l}344.80 \\
344.78 \\
345.03 \\
345.19 \\
344.69\end{array}$ \\
\hline
\end{tabular}


WHC-EP-0394-5

June 1992 Water Level Measurement Data

(Sheet 12 of 21)

\begin{tabular}{|c|c|c|c|c|}
\hline \multirow{2}{*}{ Well } & \multirow{2}{*}{ Notes } & \multirow{2}{*}{$\begin{array}{l}\text { Depth to } \\
\text { Water, ft }\end{array}$} & \multicolumn{2}{|c|}{ Elevation, ft above msl } \\
\hline & & & Adjusted Casing & Water Level \\
\hline $\begin{array}{l}3-1-16 B \\
3-1-16 C \\
3-1-17 A \\
3-1-17 B \\
3-1-17 C\end{array}$ & $\begin{array}{l}N \\
N \\
N \\
P\end{array}$ & $\begin{array}{r}35.83 \\
36.21 \\
32.75 \\
33.00 \\
1.56\end{array}$ & $\begin{array}{l}381.14 \\
382.29 \\
377.47 \\
377.87 \\
378.13\end{array}$ & $\begin{array}{l}345.31 \\
346.08 \\
344.72 \\
344.87 \\
376.57\end{array}$ \\
\hline $\begin{array}{l}3-1-18 A \\
3-1-18 B \\
3-1-18 C \\
3-1-19 \\
3-1-2\end{array}$ & $\begin{array}{l}N \\
N\end{array}$ & $\begin{array}{l}45.36 \\
44.22 \\
41.89 \\
29.80 \\
42.32\end{array}$ & $\begin{array}{l}390.83 \\
389.94 \\
388.05 \\
374.64 \\
387.02\end{array}$ & $\begin{array}{l}345.47 \\
345.72 \\
346.16 \\
344.84 \\
344.70\end{array}$ \\
\hline $\begin{array}{l}3-1-3 \\
3-1-4 \\
3-1-5 \\
3-1-6 \\
3-1-7\end{array}$ & & $\begin{array}{l}40.00 \\
35.55 \\
34.99 \\
28.56 \\
40.94\end{array}$ & $\begin{array}{l}384.73 \\
380.83 \\
379.82 \\
373.81 \\
385.63\end{array}$ & $\begin{array}{l}344.73 \\
345.28 \\
344.83 \\
345.25 \\
344.69\end{array}$ \\
\hline $\begin{array}{l}3-1-8 \\
3-1-9 \\
3-2-1 \\
3-2-2 \\
3-2-3\end{array}$ & $\begin{array}{l}N \\
Q \\
N \\
N\end{array}$ & $\begin{array}{l}40.21 \\
23.52 \\
30.57 \\
30.80 \\
30.77\end{array}$ & $\begin{array}{l}384.91 \\
384.80 \\
375.30 \\
375.53 \\
375.44\end{array}$ & $\begin{array}{l}344.70 \\
361.28 \\
344.73 \\
344.73 \\
344.67\end{array}$ \\
\hline $\begin{array}{l}3-3-1 \\
3-3-10 \\
3-3-12 \\
3-3-6 \\
3-3-7\end{array}$ & & $\begin{array}{l}39.84 \\
40.97 \\
43.89 \\
48.38 \\
49.75\end{array}$ & $\begin{array}{l}384.43 \\
385.40 \\
388.10 \\
392.85 \\
394.22\end{array}$ & $\begin{array}{l}344.59 \\
344.43 \\
344.21 \\
344.47 \\
344.47\end{array}$ \\
\hline $\begin{array}{l}3-3-9 \\
3-4-1 \\
3-4-10 \\
3-4-11 \\
3-4-7\end{array}$ & & $\begin{array}{l}43.58 \\
51.28 \\
33.17 \\
60.07 \\
34.26\end{array}$ & $\begin{array}{l}388.17 \\
395.68 \\
377.59 \\
404.53 \\
378.64\end{array}$ & $\begin{array}{l}344.59 \\
344.40 \\
344.42 \\
344.46 \\
344.38\end{array}$ \\
\hline $\begin{array}{l}3-4-9 \\
3-5-1 \\
3-6-1 \\
3-8-1 \\
3-8-2\end{array}$ & $N$ & $\begin{array}{l}37.72 \\
51.17 \\
44.32 \\
51.58 \\
53.44\end{array}$ & $\begin{array}{l}382.24 \\
395.60 \\
388.84 \\
396.20 \\
398.09\end{array}$ & $\begin{array}{l}344.52 \\
344.43 \\
344.52 \\
344.62 \\
344.65\end{array}$ \\
\hline
\end{tabular}


June 1992 Water Level Measurement Data (Sheet 13 of 21)

\begin{tabular}{|c|c|c|c|c|}
\hline \multirow{2}{*}{ Well } & \multirow{2}{*}{ Notes } & \multirow{2}{*}{$\begin{array}{l}\text { Depth to } \\
\text { Water, ft }\end{array}$} & \multicolumn{2}{|c|}{ Elevation, ft above msl } \\
\hline & & & Adjusted Casing & Water Level \\
\hline $\begin{array}{l}3-8-3 \\
30-45-16 \\
30-47-18 B \\
6-10-54 A \\
6-10-E 12\end{array}$ & N & $\begin{array}{r}50.01 \\
57.32 \\
30.68 \\
103.08 \\
73.42\end{array}$ & $\begin{array}{l}394.82 \\
404.55 \\
374.95 \\
516.40 \\
430.86\end{array}$ & $\begin{array}{l}344.23 \\
347.23 \\
344.27 \\
413.32 \\
357.44\end{array}$ \\
\hline $\begin{array}{l}6-101-48 B \\
6-11-45 A \\
6-14-38 \\
6-14-47 \\
6-15-15 A\end{array}$ & & $\begin{array}{r}11.06 \\
166.48 \\
116.08 \\
175.29 \\
148.76\end{array}$ & $\begin{array}{l}390.15 \\
578.58 \\
514.89 \\
587.23 \\
547.14\end{array}$ & $\begin{array}{l}379.09 \\
412.10 \\
404.85 \\
411.94 \\
398.38\end{array}$ \\
\hline $\begin{array}{l}6-15-26 \\
6-17-5 \\
6-17-70 \\
6-19-43 \\
6-19-58\end{array}$ & $N$ & $\begin{array}{r}123.19 \\
45.09 \\
88.73 \\
147.95 \\
154.25\end{array}$ & $\begin{array}{l}523.83 \\
433.19 \\
563.18 \\
551.58 \\
573.05\end{array}$ & $\begin{array}{l}400.64 \\
388.10 \\
474.45 \\
403.63 \\
418.80\end{array}$ \\
\hline $\begin{array}{l}6-19-88 \\
6-2-3 \\
6-2-33 A \\
6-20-20 \\
6-20-39\end{array}$ & $\begin{array}{l}N \\
N\end{array}$ & $\begin{array}{r}130.76 \\
86.68 \\
132.36 \\
104.92 \\
137.50\end{array}$ & $\begin{array}{l}644.45 \\
477.14 \\
536.37 \\
505.58 \\
539.98\end{array}$ & $\begin{array}{l}513.69 \\
390.46 \\
404.01 \\
400.66 \\
402.48\end{array}$ \\
\hline $\begin{array}{l}6-20-E 12 \\
6-21-17 \\
6-23-34 \\
6-24-1 T \\
6-24-33\end{array}$ & $N$ & $\begin{array}{r}79.48 \\
130.45 \\
130.75 \\
99.59 \\
122.19\end{array}$ & $\begin{array}{l}437.25 \\
527.31 \\
532.86 \\
475.54 \\
524.21\end{array}$ & $\begin{array}{l}357.77 \\
396.86 \\
402.11 \\
375.95 \\
402.02\end{array}$ \\
\hline $\begin{array}{l}6-24-34 A \\
6-24-34 B \\
6-24-34 C \\
6-24-35 \\
6-25-33 A\end{array}$ & N & $\begin{array}{l}131.76 \\
131.34 \\
130.44 \\
136.66 \\
126.42\end{array}$ & $\begin{array}{l}533.89 \\
533.50 \\
532.58 \\
538.81 \\
528.55\end{array}$ & $\begin{array}{l}402.13 \\
402.16 \\
402.14 \\
402.15 \\
402.13\end{array}$ \\
\hline $\begin{array}{l}6-25-34 A \\
6-25-34 B \\
6-25-34 C \\
6-26-15 A \\
6-26-33\end{array}$ & N & $\begin{array}{r}127.68 \\
126.74 \\
133.30 \\
44.68 \\
133.38\end{array}$ & $\begin{array}{l}530.01 \\
529.13 \\
535.46 \\
442.64 \\
535.49\end{array}$ & $\begin{array}{l}402.33 \\
402.39 \\
402.16 \\
397.96 \\
402.11\end{array}$ \\
\hline
\end{tabular}


WHC-EP-0394-5

June 1992 Water Level Measurement Data

(Sheet 14 of 21)

\begin{tabular}{|c|c|c|c|c|}
\hline \multirow{2}{*}{ Well } & \multirow{2}{*}{ Notes } & \multirow{2}{*}{$\begin{array}{l}\text { Depth to } \\
\text { Water, ft }\end{array}$} & \multicolumn{2}{|c|}{ Elevation, $\mathrm{ft}$ above msl } \\
\hline & & & Adjusted Casing & Water Level \\
\hline $\begin{array}{l}6-26-34 \\
6-26-35 A \\
6-26-35 C \\
6-26-89 \\
6-27-8\end{array}$ & $\begin{array}{l}N \\
N\end{array}$ & $\begin{array}{r}125.70 \\
130.06 \\
129.43 \\
181.43 \\
71.93\end{array}$ & $\begin{array}{l}528.09 \\
532.37 \\
532.13 \\
653.08 \\
465.67\end{array}$ & $\begin{array}{l}402.39 \\
402.31 \\
402.70 \\
471.65 \\
393.74\end{array}$ \\
\hline $\begin{array}{l}6-28-40 \\
6-29-70 A P \\
6-29-70 A Q \\
6-29-70 C P \\
6-29-70 C Q\end{array}$ & $\begin{array}{l}N \\
P \\
P \\
P\end{array}$ & $\begin{array}{l}156.99 \\
190.52 \\
183.50 \\
229.44 \\
232.48\end{array}$ & $\begin{array}{l}559.44 \\
629.75 \\
630.15 \\
630.94 \\
630.99\end{array}$ & $\begin{array}{l}402.45 \\
439.23 \\
446.65 \\
401.50 \\
398.51\end{array}$ \\
\hline $\begin{array}{l}6-29-70 C R \\
6-29-70 C S \\
6-29-70 C T \\
6-29-70 C U \\
6-3-45\end{array}$ & $\begin{array}{l}P \\
P \\
P \\
P\end{array}$ & $\begin{array}{r}230.64 \\
233.40 \\
234.28 \\
235.36 \\
92.52\end{array}$ & $\begin{array}{l}631.15 \\
631.31 \\
631.48 \\
631.64 \\
504.54\end{array}$ & $\begin{array}{l}400.51 \\
397.91 \\
397.20 \\
396.28 \\
412.02\end{array}$ \\
\hline $\begin{array}{l}6-31-31 \\
6-31-84 B \\
6-32-22 \\
6-34-39 A \\
6-34-51\end{array}$ & N & $\begin{array}{l}127.30 \\
203.76 \\
117.35 \\
134.54 \\
333.57\end{array}$ & $\begin{array}{l}529.32 \\
625.12 \\
517.55 \\
537.07 \\
736.76\end{array}$ & $\begin{array}{l}402.02 \\
421.36 \\
400.20 \\
402.53 \\
403.19\end{array}$ \\
\hline $\begin{array}{l}6-34-88 \\
6-35-66 \\
6-35-70 \\
6-35-78 A \\
6-35-9\end{array}$ & & $\begin{array}{l}164.33 \\
287.20 \\
243.61 \\
196.07 \\
114.29\end{array}$ & $\begin{array}{l}632.82 \\
725.65 \\
693.72 \\
660.65 \\
499.83\end{array}$ & $\begin{array}{l}468.49 \\
438.45 \\
450.11 \\
464.58 \\
385.54\end{array}$ \\
\hline $\begin{array}{l}6-36-61 A \\
6-36-93 \\
6-37-43 \\
6-37-82 A \\
6-38-65\end{array}$ & & $\begin{array}{l}340.33 \\
173.69 \\
286.92 \\
170.74 \\
323.98\end{array}$ & $\begin{array}{l}748.11 \\
644.77 \\
690.17 \\
636.75 \\
753.33\end{array}$ & $\begin{array}{l}407.78 \\
471.08 \\
403.25 \\
466.01 \\
429.35\end{array}$ \\
\hline $\begin{array}{l}6-38-70 \\
6-39-39 \\
6-39-79 \\
6-40-1 \\
6-40-33 A\end{array}$ & & $\begin{array}{r}258.07 \\
125.37 \\
208.25 \\
75.75 \\
107.24\end{array}$ & $\begin{array}{l}710.67 \\
536.65 \\
673.52 \\
438.71 \\
518.05\end{array}$ & $\begin{array}{l}452.60 \\
411.28 \\
465.27 \\
362.96 \\
410.81\end{array}$ \\
\hline
\end{tabular}


June 1992 Water Level Measurement Data (Sheet 15 of 21)

\begin{tabular}{|c|c|c|c|c|}
\hline \multirow{2}{*}{ We11 } & \multirow{2}{*}{ Notes } & \multirow{2}{*}{$\begin{array}{l}\text { Depth to } \\
\text { Water, ft }\end{array}$} & \multicolumn{2}{|c|}{ Elevation, ft above msl } \\
\hline & & & Adjusted Casing & Water Level \\
\hline $\begin{array}{l}6-40-39 \\
6-40-40 A \\
6-40-40 B \\
6-40-62 \\
6-41-23\end{array}$ & $\begin{array}{l}N \\
N\end{array}$ & $\begin{array}{r}129.04 \\
129.74 \\
130.41 \\
342.52 \\
68.99\end{array}$ & $\begin{array}{l}541.84 \\
541.00 \\
541.97 \\
747.78 \\
466.50\end{array}$ & $\begin{array}{l}412.80 \\
411.26 \\
411.56 \\
405.26 \\
397.51\end{array}$ \\
\hline $\begin{array}{l}6-41-40 \\
6-42-12 A \\
6-42-39 A \\
6-42-39 B \\
6-42-40 A\end{array}$ & $\begin{array}{l}N \\
N\end{array}$ & $\begin{array}{l}130.80 \\
138.71 \\
139.14 \\
140.05 \\
124.90\end{array}$ & $\begin{array}{l}545.94 \\
514.27 \\
557.93 \\
558.11 \\
545.53\end{array}$ & $\begin{array}{l}415.14 \\
375.56 \\
418.79 \\
418.06 \\
420.63\end{array}$ \\
\hline $\begin{array}{l}6-42-40 B \\
6-42-40 C \\
6-42-41 \\
6-42-42 B \\
6-43-104\end{array}$ & $P$ & $\begin{array}{l}125.65 \\
132.78 \\
147.30 \\
166.69 \\
269.20\end{array}$ & $\begin{array}{l}546.46 \\
546.16 \\
567.07 \\
583.23 \\
766.07\end{array}$ & $\begin{array}{l}420.81 \\
413.38 \\
419.77 \\
416.54 \\
496.87\end{array}$ \\
\hline $\begin{array}{l}6-43-40 \\
6-43-41 E \\
6-43-41 F \\
6-43-41 G \\
6-43-42\end{array}$ & $N$ & $\begin{array}{l}123.01 \\
130.83 \\
130.93 \\
135.60 \\
146.12\end{array}$ & $\begin{array}{l}541.99 \\
550.86 \\
551.01 \\
551.13 \\
566.36\end{array}$ & $\begin{array}{l}418.98 \\
420.03 \\
420.08 \\
415.53 \\
420.24\end{array}$ \\
\hline $\begin{array}{l}6-43-42 J \\
6-43-43 \\
6-43-45 \\
6-43-89 \\
6-43-91 A P\end{array}$ & N & $\begin{array}{l}163.52 \\
164.52 \\
193.91 \\
178.44 \\
230.80\end{array}$ & $\begin{array}{l}581.68 \\
579.37 \\
597.68 \\
644.15 \\
671.51\end{array}$ & $\begin{array}{l}418.16 \\
414.85 \\
403.77 \\
465.71 \\
440.71\end{array}$ \\
\hline $\begin{array}{l}6-43-91 A Q \\
6-43-910 \\
6-44-42 \\
6-44-43 B \\
6-44-64\end{array}$ & $\begin{array}{l}N \\
N\end{array}$ & $\begin{array}{l}230.78 \\
261.02 \\
158.96 \\
164.77 \\
319.08\end{array}$ & $\begin{array}{l}671.94 \\
671.99 \\
579.22 \\
580.12 \\
725.60\end{array}$ & $\begin{array}{l}441.16 \\
410.97 \\
420.26 \\
415.35 \\
406.52\end{array}$ \\
\hline $\begin{array}{l}6-44-91 P \\
6-44-91 Q \\
6-44-91 R \\
6-44-91 S \\
6-44-91 T\end{array}$ & $\begin{array}{l}P \\
P \\
P \\
P \\
P\end{array}$ & $\begin{array}{l}267.40 \\
267.70 \\
266.42 \\
273.78 \\
274.85\end{array}$ & $\begin{array}{l}672.15 \\
672.33 \\
672.49 \\
672.68 \\
672.84\end{array}$ & $\begin{array}{l}404.75 \\
404.63 \\
406.07 \\
398.90 \\
397.99\end{array}$ \\
\hline
\end{tabular}


June 1992 Water Level Measurement Data (Sheet 16 of 21)

\begin{tabular}{|c|c|c|c|c|}
\hline \multirow{2}{*}{ Well } & \multirow{2}{*}{ Notes } & \multirow{2}{*}{$\begin{array}{l}\text { Depth to } \\
\text { Water, ft }\end{array}$} & \multicolumn{2}{|c|}{ Elevation, $\mathrm{ft}$ above msl } \\
\hline & & & Adjusted Casing & Water Level \\
\hline $\begin{array}{l}6-44-91 U \\
6-45-42 \\
6-45-69 A \\
6-46-21 B \\
6-47-35 A\end{array}$ & $\begin{array}{l}P \\
N \\
N \\
N\end{array}$ & $\begin{array}{r}275.17 \\
160.98 \\
278.25 \\
131.04 \\
62.69\end{array}$ & $\begin{array}{l}673.01 \\
577.33 \\
725.46 \\
522.02 \\
476.36\end{array}$ & $\begin{array}{l}397.84 \\
416.35 \\
447.21 \\
390.98 \\
413.67\end{array}$ \\
\hline $\begin{array}{l}6-47-35 B \\
6-47-46 A \\
6-47-50 \\
6-47-60 \\
6-47-80 A P\end{array}$ & $\mathrm{~N}$ & $\begin{array}{r}63.02 \\
175.81 \\
180.05 \\
249.08 \\
271.50\end{array}$ & $\begin{array}{l}476.65 \\
580.14 \\
583.87 \\
649.84 \\
713.03\end{array}$ & $\begin{array}{l}413.63 \\
404.33 \\
403.82 \\
400.76 \\
441.53\end{array}$ \\
\hline $\begin{array}{l}6-47-80 A Q \\
6-47-80 C P \\
6-47-80 C Q \\
6-47-80 C R \\
6-47-80 C S\end{array}$ & $\begin{array}{l}N \\
N \\
N \\
N \\
N\end{array}$ & $\begin{array}{l}261.80 \\
307.18 \\
308.03 \\
308.03 \\
314.00\end{array}$ & $\begin{array}{l}713.51 \\
712.58 \\
712.75 \\
712.92 \\
713.10\end{array}$ & $\begin{array}{l}451.71 \\
405.40 \\
404.72 \\
404.89 \\
399.10\end{array}$ \\
\hline $\begin{array}{l}6-47-80 \mathrm{CT} \\
6-47-80 \mathrm{CU} \\
6-48-50 \\
6-48-7 \\
6-48-71\end{array}$ & $\begin{array}{l}N \\
N \\
N \\
N\end{array}$ & $\begin{array}{r}314.37 \\
314.56 \\
169.88 \\
27.09 \\
242.92\end{array}$ & $\begin{array}{l}713.25 \\
713.43 \\
574.06 \\
384.72 \\
688.15\end{array}$ & $\begin{array}{l}398.88 \\
398.87 \\
404.18 \\
357.63 \\
445.23\end{array}$ \\
\hline $\begin{array}{l}6-49-13 E \\
6-49-28 \\
6-49-55 A \\
6-49-55 B \\
6-49-57 A\end{array}$ & $\begin{array}{l}P \\
N\end{array}$ & $\begin{array}{r}51.45 \\
141.45 \\
128.42 \\
128.51 \\
151.03\end{array}$ & $\begin{array}{l}412.72 \\
535.40 \\
530.14 \\
530.33 \\
552.81\end{array}$ & $\begin{array}{l}361.27 \\
393.95 \\
401.72 \\
401.82 \\
401.78\end{array}$ \\
\hline $\begin{array}{l}6-49-57 B \\
6-49-79 \\
6-50-28 B \\
6-50-30 \\
6-50-42\end{array}$ & $N$ & $\begin{array}{r}153.52 \\
233.13 \\
143.46 \\
134.58 \\
55.96\end{array}$ & $\begin{array}{l}555.99 \\
689.20 \\
537.3 \mathrm{~J} \\
528.84 \\
466.84\end{array}$ & $\begin{array}{l}402.47 \\
456.07 \\
393.84 \\
394.26 \\
410.88\end{array}$ \\
\hline $\begin{array}{l}6-50-45 \\
6-50-48 B \\
6-50-53 A \\
6-50-53 B \\
6-50-85\end{array}$ & $\begin{array}{l}P \\
N \\
N \\
N\end{array}$ & $\begin{array}{r}42.90 \\
144.42 \\
154.91 \\
154.76 \\
284.07\end{array}$ & $\begin{array}{l}451.41 \\
550.39 \\
556.30 \\
557.62 \\
739.35\end{array}$ & $\begin{array}{l}408.51 \\
405.97 \\
401.39 \\
402.86 \\
455.28\end{array}$ \\
\hline
\end{tabular}


June 1992 Water Level Measurement Data (Sheet 17 of 21)

\begin{tabular}{|c|c|c|c|c|}
\hline \multirow{2}{*}{ Well } & \multirow{2}{*}{ Notes } & \multirow{2}{*}{$\begin{array}{l}\text { Depth to } \\
\text { Water, ft }\end{array}$} & \multicolumn{2}{|c|}{ Elevation, $\mathrm{ft}$ above $\mathrm{ms}$} \\
\hline & & & Adjusted Casing & Water Level \\
\hline $\begin{array}{l}6-51-46 \\
6-51-63 \\
6-51-75 \\
6-52-19 \\
6-52-46 A\end{array}$ & $P$ & $\begin{array}{r}37.64 \\
167.00 \\
192.32 \\
49.45 \\
47.03\end{array}$ & $\begin{array}{l}444.63 \\
571.84 \\
641.51 \\
411.08 \\
455.61\end{array}$ & $\begin{array}{l}406.99 \\
404.84 \\
449.19 \\
361.63 \\
408.58\end{array}$ \\
\hline $\begin{array}{l}6-52-48 \\
6-52-54 \\
6-52-57 \\
6-53-35 \\
6-53-47 A\end{array}$ & $\begin{array}{l}P \\
N \\
N\end{array}$ & $\begin{array}{r}61.25 \\
166.20 \\
159.74 \\
131.24 \\
32.62\end{array}$ & $\begin{array}{l}466.06 \\
530.99 \\
438.28\end{array}$ & $\begin{array}{l}404.81 \\
399.75 \\
405.66\end{array}$ \\
\hline $\begin{array}{l}6-53-47 B \\
6-53-48 A \\
6-53-48 B \\
6-53-50 \\
6-53-55 A\end{array}$ & $P$ & $\begin{array}{r}32.93 \\
39.12 \\
38.65 \\
39.88 \\
174.55\end{array}$ & $\begin{array}{l}438.58 \\
442.45 \\
442.71 \\
444.21 \\
575.63\end{array}$ & $\begin{array}{l}405.65 \\
403.33 \\
404.06 \\
404.33 \\
401.08\end{array}$ \\
\hline $\begin{array}{l}6-53-55 B \\
6-53-55 C \\
6-54-19 \\
6-54-34 \\
6-54-42\end{array}$ & $\begin{array}{l}P \\
N \\
N\end{array}$ & $\begin{array}{r}175.13 \\
174.15 \\
21.77 \\
140.36 \\
115.31\end{array}$ & $\begin{array}{l}576.16 \\
576.08 \\
383.60 \\
550.24 \\
511.49\end{array}$ & $\begin{array}{l}401.03 \\
401.93 \\
361.83 \\
409.88 \\
396.18\end{array}$ \\
\hline $\begin{array}{l}6-54-45 A \\
6-54-48 \\
6-54-57 \\
6-55-21 \\
6-55-40\end{array}$ & $P$ & $\begin{array}{r}96.37 \\
55.02 \\
174.14 \\
35.63 \\
133.66\end{array}$ & $\begin{array}{l}494.29 \\
457.02 \\
575.58 \\
395.96 \\
543.13\end{array}$ & $\begin{array}{l}397.92 \\
402.00 \\
401.44 \\
360.33 \\
409.47\end{array}$ \\
\hline $\begin{array}{l}6-55-44 \\
6-55-50 c \\
6-55-55 \\
6-55-57 \\
6-55-70\end{array}$ & $N$ & $\begin{array}{r}124.04 \\
42.43 \\
161.91 \\
166.23 \\
136.84\end{array}$ & $\begin{array}{c}519.67 \\
444.43 \\
\mathrm{a} \\
566.66 \\
569.03\end{array}$ & $\begin{array}{l}395.63 \\
402.00 \\
400.43 \\
432.19\end{array}$ \\
\hline $\begin{array}{l}6-55-76 \\
6-55-89 \\
6-55-95 \\
6-56-43 \\
6-56-53\end{array}$ & $\begin{array}{l}N \\
P\end{array}$ & $\begin{array}{r}139.19 \\
163.11 \\
311.78 \\
131.74 \\
31.80\end{array}$ & $\begin{array}{l}583.24 \\
617.43 \\
777.05 \\
540.42 \\
434.34\end{array}$ & $\begin{array}{l}444.05 \\
454.32 \\
465.27 \\
408.68 \\
402.54\end{array}$ \\
\hline
\end{tabular}


June 1992 Water Level Measurement Data (Sheet 18 of 21)

\begin{tabular}{|c|c|c|c|c|}
\hline \multirow{2}{*}{ Well } & \multirow{2}{*}{ Notes } & \multirow{2}{*}{$\begin{array}{l}\text { Depth to } \\
\text { Water, ft }\end{array}$} & \multicolumn{2}{|c|}{ Elevation, ft above msl } \\
\hline & & & Adjusted Casing & Water Level \\
\hline $\begin{array}{l}6-57-25 A \\
6-57-29 A \\
6-57-29 B \\
6-57-83 A \\
6-58-24\end{array}$ & $\begin{array}{l}N \\
Q\end{array}$ & $\begin{array}{r}50.53 \\
53.68 \\
54.39 \\
145.12 \\
57.09\end{array}$ & $\begin{array}{l}414.57 \\
408.47 \\
416.18 \\
577.96 \\
418.80\end{array}$ & $\begin{array}{l}364.04 \\
354.79 \\
361.79 \\
432.84 \\
361.71\end{array}$ \\
\hline $\begin{array}{l}6-59-32 \\
6-59-58 \\
6-59-80 B \\
6-60-32 \\
6-60-57\end{array}$ & & $\begin{array}{r}62.18 \\
96.02 \\
153.70 \\
63.39 \\
67.54\end{array}$ & $\begin{array}{l}424.29 \\
497.77 \\
583.25 \\
425.30 \\
469.64\end{array}$ & $\begin{array}{l}362.11 \\
401.75 \\
429.55 \\
361.91 \\
402.10\end{array}$ \\
\hline $\begin{array}{l}6-60-60 \\
6-61-37 \\
6-61-41 \\
6-61-62 \\
6-61-66\end{array}$ & & $\begin{array}{r}110.23 \\
61.18 \\
33.00 \\
95.74 \\
121.18\end{array}$ & $\begin{array}{l}512.03 \\
442.94 \\
428.92 \\
497.51 \\
522.18\end{array}$ & $\begin{array}{l}401.80 \\
381.76 \\
395.92 \\
401.77 \\
401.00\end{array}$ \\
\hline $\begin{array}{l}6-62-31 \\
6-62-43 A \\
6-63-25 A \\
6-63-51 \\
6-63-55\end{array}$ & & $\begin{array}{l}72.35 \\
36.07 \\
33.49 \\
24.85 \\
26.15\end{array}$ & $\begin{array}{l}434.12 \\
432.30 \\
395.15 \\
424.54 \\
426.54\end{array}$ & $\begin{array}{l}361.77 \\
396.23 \\
361.66 \\
399.69 \\
400.39\end{array}$ \\
\hline $\begin{array}{l}6-63-58 \\
6-63-90 \\
6-63-92 \\
6-64-27 \\
6-64-62\end{array}$ & $\begin{array}{l}N \\
N\end{array}$ & $\begin{array}{r}91.18 \\
110.64 \\
97.01 \\
52.40 \\
99.75\end{array}$ & $\begin{array}{l}491.90 \\
509.73 \\
497.50 \\
414.29 \\
500.25\end{array}$ & $\begin{array}{l}400.72 \\
399.09 \\
400.49 \\
361.89 \\
400.50\end{array}$ \\
\hline $\begin{array}{l}6-65-22 \\
6-65-23 \\
6-65-23 \\
6-65-38 \\
6-65-50\end{array}$ & & $\begin{array}{l}27.64 \\
26.14 \\
25.50 \\
32.50 \\
67.38\end{array}$ & $\begin{array}{l}391.10 \\
387.93 \\
387.93 \\
433.58 \\
467.06\end{array}$ & $\begin{array}{l}363.46 \\
361.79 \\
362.43 \\
401.08 \\
399.68\end{array}$ \\
\hline $\begin{array}{l}6-65-59 A \\
6-65-72 \\
6-65-83 \\
6-65-95 \\
6-66-103\end{array}$ & $N$ & $\begin{array}{r}106.56 \\
141.34 \\
87.23 \\
52.17 \\
64.23\end{array}$ & $\begin{array}{l}506.96 \\
540.28 \\
485.63 \\
452.26 \\
463.01\end{array}$ & $\begin{array}{l}400.40 \\
398.94 \\
398.40 \\
400.09 \\
398.78\end{array}$ \\
\hline
\end{tabular}


WHC-EP-0394-5

June 1992 Water Level Measurement Data

(Sheet 19 of 21)

\begin{tabular}{|c|c|c|c|c|}
\hline \multirow{2}{*}{ Well } & \multirow{2}{*}{ Notes } & \multirow{2}{*}{$\begin{array}{l}\text { Depth to } \\
\text { Water, ft }\end{array}$} & \multicolumn{2}{|c|}{ Elevation, $\mathrm{ft}$ above msl } \\
\hline & & & Adjusted Casing & Water Leve1 \\
\hline $\begin{array}{l}6-66-38 \\
6-66-39 \\
6-66-58 \\
6-66-64 \\
6-66-91\end{array}$ & $N$ & $\begin{array}{r}33.76 \\
47.15 \\
103.00 \\
105.98 \\
67.85\end{array}$ & $\begin{array}{l}436.24 \\
453.78 \\
503.33 \\
505.92 \\
467.75\end{array}$ & $\begin{array}{l}402.48 \\
406.63 \\
400.33 \\
399.94 \\
399.90\end{array}$ \\
\hline $\begin{array}{l}6-67-51 \\
6-67-86 \\
6-67-98 \\
6-68-105 \\
6-69-38\end{array}$ & & $\begin{array}{r}124.82 \\
74.03 \\
55.68 \\
54.80 \\
22.15\end{array}$ & $\begin{array}{l}524.59 \\
472.39 \\
455.47 \\
451.85 \\
424.10\end{array}$ & $\begin{array}{l}399.77 \\
398.36 \\
399.79 \\
397.05 \\
401.95\end{array}$ \\
\hline $\begin{array}{l}6-69-450 \\
6-70-23 \\
6-70-68 \\
6-71-30 \\
6-71-52\end{array}$ & & $\begin{array}{r}88.09 \\
27.60 \\
127.14 \\
30.44 \\
123.82\end{array}$ & $\begin{array}{l}487.18 \\
391.71 \\
526.21 \\
400.68 \\
523.04\end{array}$ & $\begin{array}{l}399.09 \\
364.11 \\
399.07 \\
370.24 \\
399.22\end{array}$ \\
\hline $\begin{array}{l}6-71-77 \\
6-72-73 \\
6-72-88 \\
6-72-92 \\
6-72-98\end{array}$ & & $\begin{array}{l}75.29 \\
85.23 \\
36.19 \\
51.64 \\
51.33\end{array}$ & $\begin{array}{l}472.28 \\
482.57 \\
437.37 \\
452.22 \\
454.19\end{array}$ & $\begin{array}{l}396.99 \\
397.34 \\
401.18 \\
400.58 \\
402.86\end{array}$ \\
\hline $\begin{array}{l}6-73-61 \\
6-74-44 \\
6-74-48 \\
6-77-36 \\
6-77-54\end{array}$ & & $\begin{array}{r}132.39 \\
48.65 \\
89.17 \\
36.42 \\
83.37\end{array}$ & $\begin{array}{l}531.53 \\
445.18 \\
487.18 \\
412.28 \\
480.59\end{array}$ & $\begin{array}{l}399.14 \\
396.53 \\
398.01 \\
375.86 \\
397.22\end{array}$ \\
\hline $\begin{array}{l}6-78-62 \\
6-8-17 \\
6-8-25 \\
6-8-32 \\
6-80-435\end{array}$ & & $\begin{array}{r}74.49 \\
124.31 \\
109.89 \\
154.50 \\
24.65\end{array}$ & $\begin{array}{l}469.88 \\
522.44 \\
509.30 \\
554.39 \\
412.52\end{array}$ & $\begin{array}{l}395.39 \\
398.13 \\
399.41 \\
399.89 \\
387.87\end{array}$ \\
\hline $\begin{array}{l}6-81-38 \\
6-81-58 \\
6-82-45 A \\
6-83-36 \\
6-83-47\end{array}$ & & $\begin{array}{l}27.22 \\
45.86 \\
24.79 \\
40.82 \\
46.45\end{array}$ & $\begin{array}{l}406.47 \\
439.55 \\
413.73 \\
418.63 \\
435.27\end{array}$ & $\begin{array}{l}379.25 \\
393.69 \\
388.94 \\
377.81 \\
388.82\end{array}$ \\
\hline
\end{tabular}


June 1992 Water Level Measurement Data (Sheet 20 of 21)

\begin{tabular}{|c|c|c|c|c|}
\hline \multirow{2}{*}{ Well } & \multirow{2}{*}{ Notes } & \multirow{2}{*}{$\begin{array}{l}\text { Depth to } \\
\text { Water, ft }\end{array}$} & \multicolumn{2}{|c|}{ Elevation, ft above msl } \\
\hline & & & Adjusted Casing & Water Level \\
\hline $\begin{array}{l}6-84-35 A \\
6-85-40 A \\
6-86-42 \\
6-87-42 A \\
6-87-55\end{array}$ & $Q$ & $\begin{array}{r}7.25 \\
28.84 \\
25.24 \\
32.54 \\
71.64\end{array}$ & $\begin{array}{l}400.05 \\
412.38 \\
409.92 \\
416.53 \\
458.63\end{array}$ & $\begin{array}{l}392.80 \\
383.54 \\
384.68 \\
383.99 \\
386.99\end{array}$ \\
\hline $\begin{array}{l}6-88-41 \\
6-89-35 \\
6-9-E 2 \\
6-90-34 \\
6-90-45\end{array}$ & & $\begin{array}{l}33.70 \\
25.72 \\
45.91 \\
20.98 \\
37.28\end{array}$ & $\begin{array}{l}416.04 \\
397.46 \\
418.09 \\
392.39 \\
422.15\end{array}$ & $\begin{array}{l}382.34 \\
371.74 \\
372.18 \\
371.41 \\
384.87\end{array}$ \\
\hline $\begin{array}{l}6-91-37 \\
6-92-49 \\
6-93-46 \\
6-93-49 B \\
6-96-49\end{array}$ & $\begin{array}{l}\mathrm{N} \\
\mathrm{N}\end{array}$ & $\begin{array}{l}48.90 \\
48.24 \\
32.89 \\
54.53 \\
36.86\end{array}$ & $\begin{array}{c}422.93 \\
432.00 \\
a \\
a \\
419.29\end{array}$ & $\begin{array}{l}374.03 \\
383.76 \\
382.43\end{array}$ \\
\hline $\begin{array}{l}6-96-52 \\
6-97-43 \\
6-97-51 A \\
6-512-29 \\
6-512-3\end{array}$ & $N$ & $\begin{array}{l}30.88 \\
42.54 \\
19.80 \\
83.39 \\
54.84\end{array}$ & $\begin{array}{c}a \\
421.81 \\
402.49 \\
487.68 \\
435.52\end{array}$ & $\begin{array}{l}379.27 \\
382.69 \\
404.29 \\
380.68\end{array}$ \\
\hline $\begin{array}{l}6-S 14-20 A \\
6-S 18-E 2 A \\
6-S 19-11 \\
6-S 19-E 13 \\
6-S 27-E 14\end{array}$ & $N$ & $\begin{array}{l}91.93 \\
75.41 \\
93.70 \\
48.67 \\
55.41\end{array}$ & $\begin{array}{l}492.74 \\
434.85 \\
483.74 \\
394.51 \\
399.76\end{array}$ & $\begin{array}{l}400.81 \\
359.44 \\
390.04 \\
345.84 \\
344.35\end{array}$ \\
\hline $\begin{array}{l}6-\$ 29-E 12 \\
6-S 3-25 \\
6-S 3-E 12 \\
6-S 30-E 10 A \\
6-S 30-E 10 B\end{array}$ & & $\begin{array}{r}41.77 \\
124.40 \\
43.50 \\
43.44 \\
42.67\end{array}$ & $\begin{array}{l}387.96 \\
523.50 \\
397.90 \\
392.29 \\
392.07\end{array}$ & $\begin{array}{l}346.19 \\
399.10 \\
354.40 \\
348.85 \\
349.40\end{array}$ \\
\hline $\begin{array}{l}6-S 30-E 15 A \\
6-\$ 31-1 \\
6-S 31-E 10 A \\
6-S 31-E 1 O B \\
6-S 31-E 1 O C\end{array}$ & $\mathbf{N}$ & $\begin{array}{l}55.84 \\
83.74 \\
36.08 \\
34.53 \\
33.81\end{array}$ & $\begin{array}{l}400.14 \\
460.00 \\
384.57 \\
383.71 \\
382.91\end{array}$ & $\begin{array}{l}344.30 \\
376.26 \\
348.49 \\
349.18 \\
349.10\end{array}$ \\
\hline
\end{tabular}


June 1992 Water Level Measirement Data (Sheet 21 of 21)

\begin{tabular}{|c|c|c|c|c|}
\hline \multirow{2}{*}{ Well } & \multirow{2}{*}{ Notes } & \multirow{2}{*}{$\begin{array}{l}\text { Depth to } \\
\text { Water, ft }\end{array}$} & \multicolumn{2}{|c|}{ Elevation, ft above msl } \\
\hline & & & Adjusted Casing & Water Level \\
\hline $\begin{array}{l}6-S 31-E 10 D \\
6-S 31-E 13 \\
6-S 31-E 8 \\
6-S 32-E 13 A \\
6-S 32-E 13 B\end{array}$ & $\begin{array}{l}N \\
N \\
N\end{array}$ & $\begin{array}{l}31.57 \\
47.15 \\
20.75 \\
43.22 \\
47.24\end{array}$ & $\begin{array}{l}380.58 \\
394.06 \\
374.75 \\
390.46 \\
394.72\end{array}$ & $\begin{array}{l}349.01 \\
346.91 \\
354.00 \\
347.24 \\
347.48\end{array}$ \\
\hline $\begin{array}{l}6-S 32-E 8 \\
6-S 34-E 10 \\
6-S 36-E 11 \\
6-S 36-E 13 A \\
6-S 37-E 11\end{array}$ & N & $\begin{array}{l}15.81 \\
29.11 \\
47.63 \\
47.95 \\
47.38\end{array}$ & $\begin{array}{l}375.50 \\
382.37 \\
399.04 \\
399.30 \\
399.30\end{array}$ & $\begin{array}{l}359.69 \\
353.26 \\
351.41 \\
351.35 \\
351.92\end{array}$ \\
\hline $\begin{array}{l}6-\$ 37-E 14 \\
6-S 38-E 12 A \\
6-\$ 38-E 12 B \\
6-\$ 40-E 14 \\
6-S 41-E 11\end{array}$ & $N$ & $\begin{array}{l}57.24 \\
53.19 \\
53.23 \\
50.30 \\
47.92\end{array}$ & $\begin{array}{l}408.28 \\
404.95 \\
405.00 \\
402.85 \\
401.36\end{array}$ & $\begin{array}{l}351.04 \\
351.76 \\
351.77 \\
352.55 \\
353.44\end{array}$ \\
\hline $\begin{array}{l}6-S 41-E 12 \\
6--541-E 13 A \\
61-S 41-E 13 B \\
6-S 41-E 13 C \\
6-S 43-E 12\end{array}$ & $\begin{array}{l}N \\
N\end{array}$ & $\begin{array}{l}49.68 \\
58.25 \\
57.78 \\
58.58 \\
52.53\end{array}$ & $\begin{array}{l}401.93 \\
410.56 \\
410.10 \\
410.67 \\
405.60\end{array}$ & $\begin{array}{l}352.25 \\
352.31 \\
352.32 \\
352.09 \\
353.07\end{array}$ \\
\hline $\begin{array}{l}6-S 6-E 14 A \\
6-S 6-E 40 \\
6-S 7-34 \\
6-S 8-19\end{array}$ & & $\begin{array}{r}27.60 \\
57.93 \\
118.94 \\
107.02\end{array}$ & $\begin{array}{l}378.29 \\
430.47 \\
527.12 \\
503.81\end{array}$ & $\begin{array}{l}350.69 \\
372.54 \\
408.18 \\
396.79\end{array}$ \\
\hline
\end{tabular}

Note : $N=$ Well not included on map.

$P=$ Well in confined aquifer, not included on map.

$Q=$ Questionable data, not included on map.

$C=$ Elevation in reference to local (100-D, 100-N) datum. Other elevations are from Hanford Wells (McGhan 1989).

$a=$ Casing elevation is currently unavailable. 
WHC-EP-0394-5

DISTRIBUTION

Number of Copies

4

U.S. Department of Energy Richland Field office

G. J. Bracken

A4-02

M. J. Furman

A5-21

A. J. Lassila

A5-10

Public Reading Room

Al-65

9

Pacific Northwest Laboratory
M. P. Bergeron
K6-77
R. W. Bryce
K6-96
M. A. Chamness
K6-96
M. D. Freshley
K6-77
G. V. Last
$\mathrm{HO}-32$
S. P. Luttrell
$\mathrm{K} 6-96$
D. R. Newcomer
$\mathrm{K} 6-96$
R. M. Smith
B1-40
F. A. Spane
K6-96

Westinghouse Hanford Company

M. R. Adams

H6-01

M. A. Buckmaster

$\mathrm{H} 6-03$

J. A. Caggiano

H6-06

L. P. Diediker

T1-30

C. D. Delaney

J. J. Dorian

H6-06

G. L. Dunford

H6-2O

D. B. Erb

J. W. Fassett

$\mathrm{R} 1-51$

K. R. Fecht

H6-03

H6-06

B. H. Ford

H6-06

M. J. Hartman

H6-06

F. D. Hodges

H6-06

D. G. Horton

H6-06

R. L. Jackson

H6-06

V. G. Johnson

H6-06

W. L. Johnson

H6-06

W. A. Jordan

H6-04

G. L. Kasza (3)

H6-06

A. J. Knepp

H6-06

M. J. Lauterbach

H6-06

A. G. Law

H6-01

H6-06 


\section{Westinghouse Hanford Company (Cont)}

K. A. Lindsey

M. J. Marratt

H6-06

R. B. Mercer

R. D. Miller

D. J. Moak

H6-06

R. E. Peterson

H6-06

S. M. Price

W. H. Price

S. P. Raidel

H6-06

N3-05

H6-06

H6-23

N3-05

R. F. Raidl

H6-06

K. D. Reynolds

H6-06

V. J. Rohay

A. L. Schatz

H6-06

J. S. Schmid

H6-06

N3-05

J. A. Serkowski

H6-06

K. R. Simpson

H6-06

K. A. Smith

H6-06

L. C. Swanson

T4-06

R. J. Thomas (2)

H6-06

R. R. Thompson

R3-12

S. J. Trent

L4-96

P. J. Valcich

D. C. Weekes

H6-06

H6-04

C. D. Wittreich

H6-06

Central Files

H6-03

EDMC (2)

L8-04

Publications Services (3)

H4-22

Technical Library

H4-16

P8-55 

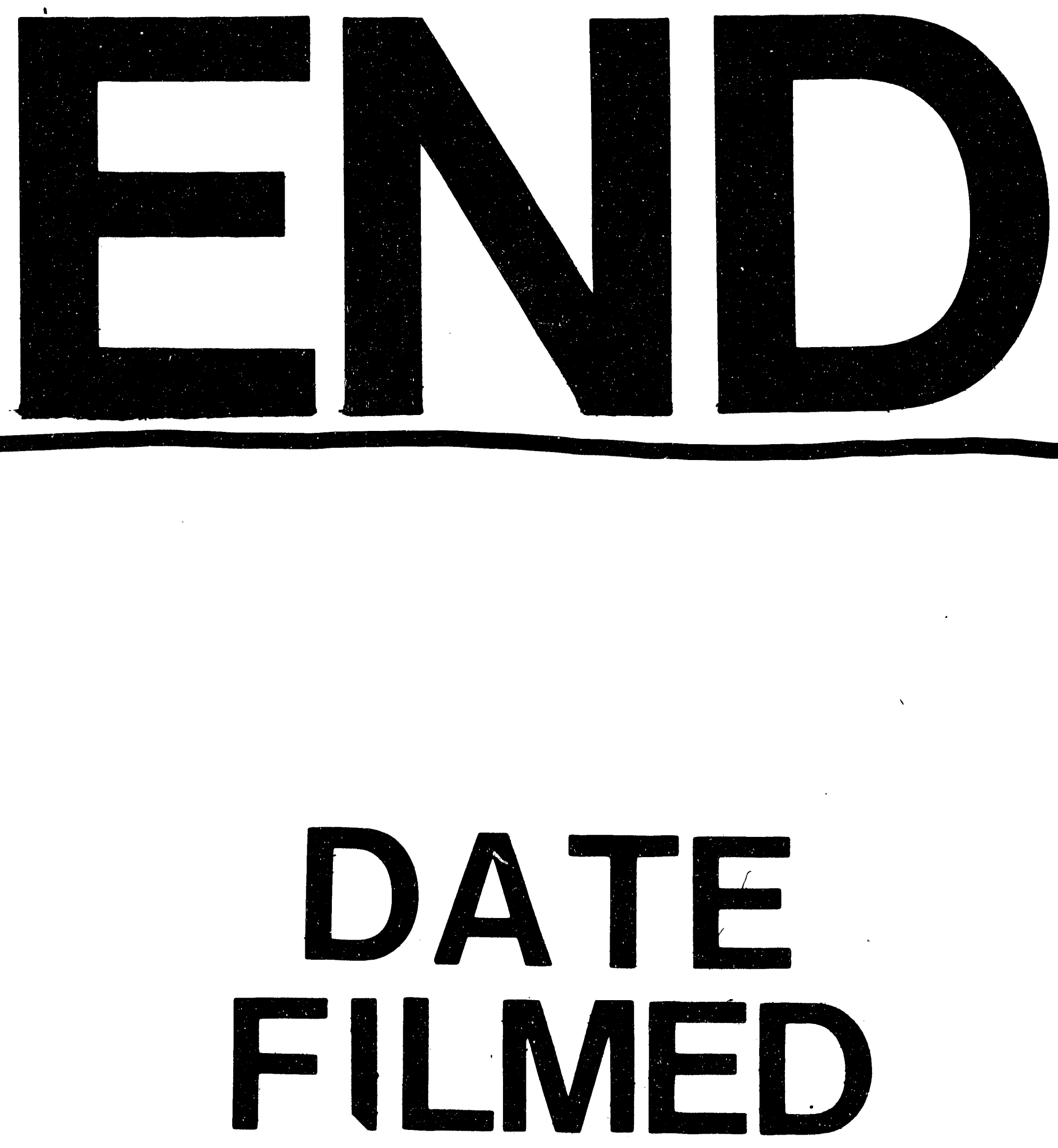

9
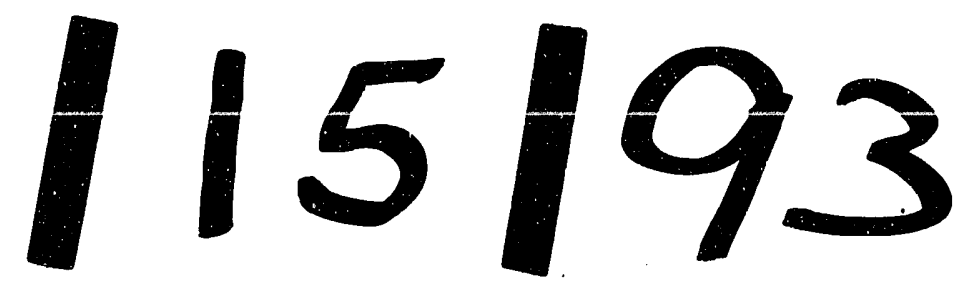
